arXiv:0704.0292 [hep-ph]

April 2007

\title{
A practical Seedless Infrared-Safe Cone jet algorithm
}

\author{
Gavin P. Salam and Grégory Soyez ${ }^{* \dagger}$ \\ LPTHE, \\ Université Pierre et Marie Curie - Paris 6, \\ Université Denis Diderot - Paris 7, \\ CNRS UMR 7589, 75252 Paris cedex 05, France.
}

\begin{abstract}
Current cone jet algorithms, widely used at hadron colliders, take event particles as seeds in an iterative search for stable cones. A longstanding infrared (IR) unsafety issue in such algorithms is often assumed to be solvable by adding extra 'midpoint' seeds, but actually is just postponed to one order higher in the coupling. A proper solution is to switch to an exact seedless cone algorithm, one that provably identifies all stable cones. The only existing approach takes $N 2^{N}$ time to find jets among $N$ particles, making it unusable at hadron level. This can be reduced to $N^{2} \ln N$ time, leading to code (SISCone) whose speed is similar to that of public midpoint implementations. Monte Carlo tests provide a strong cross-check of an analytical proof of the IR safety of the new algorithm, and the absence of any ' $R_{\text {sep }}$ ' issue implies a good practical correspondence between parton and hadron levels. Relative to a midpoint cone, the use of an IR safe seedless algorithm leads to modest changes for inclusive jet spectra, mostly through reduced sensitivity to the underlying event, and significant changes for some multi-jet observables.
\end{abstract}

SISCone, the C++ implementation of the algorithm, is available at

http://projects.hepforge.org/siscone/ (standalone), http://www.lpthe.jussieu.fr/ salam/fastjet/ (FastJet plugin).

${ }^{*}$ On leave from the PTF group of the University of Liège.

${ }^{\dagger}$ Current address: Physics Department, Brookhaven National Laboratory, Upton, NY 11973, USA 


\section{Contents}

1 Introduction $\quad 2$

2 Overview of the cone jet-finding algorithm

3 IR unsafety in the midpoint algorithm

4 An exact seedless cone jet definition 8

4.1 One-dimensional example . . . . . . . . . . . . . . . . . . . . . . . 9

4.2 The two-dimensional case . . . . . . . . . . . . . . . . 10

4.2 .1 General approach . . . . . . . . . . . . . . . . 10

4.2.2 Specific computational strategies . . . . . . . . . . . . . 11

4.3 The split-merge part of the cone algorithm . . . . . . . . . . . . . 14

5 Tests and comparisons $\quad \mathbf{1 6}$

5.1 Measures of IR (un)safety . . . . . . . . . . . . . . . . . . . . . . . . . . . .

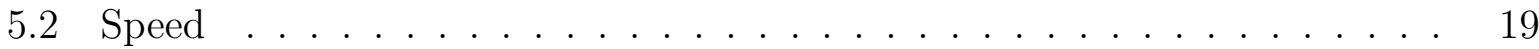

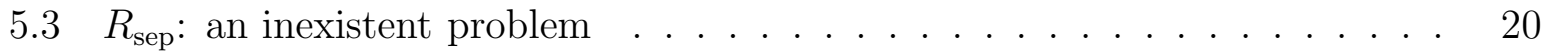

5.4 Physics impact of seedless v. midpoint cone . . . . . . . . . . . . . . . 23

5.4 .1 Inclusive jet spectrum . . . . . . . . . . . . . . . . 24

5.4 .2 Jet masses in 3 -jet events . . . . . . . . . . . . . . . 26

6 Conclusions $\quad 30$

A Further computational details $\quad 32$

A.1 Cone multiplicities . . . . . . . . . . . . . . . . . . . . . 32

A.2 Computational complexity of the split-merge step . . . . . . . . . . . 32

B Proof of IR safety of the SISCone algorithm

B.1 General aspects of the proof . . . . . . . . . . . . . . 34

B.2 Split-merge ordering variable . . . . . . . . . . . . . . 37

\section{Introduction}

Two broad classes of jet definition are generally advocated [1] for hadron colliders. One option is to use sequential recombination jet algorithms, such as the $k_{t}$ [2] and Cambridge/Aachen algorithms [3], which introduce a distance measure between particles, and repeatedly recombine the closest pair of particles until some stopping criterion is reached. While experimentally these are starting to be investigated [4, 5], the bulk of measurements are currently carried out with the other class of jet definition, cone jet algorithms (see e.g. [6]). In general there are indications [7] that it may be advantageous to use both sequential 
recombination and cone jet algorithms because of complementary sensitivities to different classes of non-perturbative corrections.

Cone jet algorithms are inspired by the idea [8] of defining a jet as an angular cone around some direction of dominant energy flow. To find these directions of dominant energy flow, cone algorithms usually take some (or all) of the event particles as 'seeds', i.e. trial cone directions. Then for each seed they establish the list of particles in the trial cone, evaluate the sum of their 4-momenta, and use the resulting 4-momentum as a new trial direction for the cone. This procedure is iterated until the cone direction no longer changes, i.e. until one has a "stable cone".

Stable cones have the property that the cone axis $a$ (a four-vector) coincides with the (four-vector) axis defined by the total momentum of the particles contained in the cone,

$$
D\left(p_{\text {in cone }}, a\right)=0, \quad \text { with } \quad p_{\text {in cone }}=\sum_{i} p_{i} \Theta\left(R-D\left(p_{i}, a\right)\right),
$$

where $D(p, a)$ is some measure of angular distance between the four-momentum $p$ and the cone axis $a$, and $R$ is the given opening (half)-angle of the cone, also referred to as the cone radius. Typically one defines $D^{2}(p, a)=\left(y_{p}-y_{a}\right)^{2}+\left(\phi_{p}-\phi_{a}\right)^{2}$, where $y_{p}, y_{a}$ and $\phi_{p}, \phi_{a}$ are respectively the rapidity and azimuth of $p$ and $a$.

Two types of problem arise when using seeds as starting points of an iterative search for stable cones. On one hand, if one only uses particles above some momentum threshold as seeds, then the procedure is collinear unsafe. Alternatively if any particle can act as a seed then one needs to be sure that the addition of an infinitely soft particle cannot lead to a new (hard) stable cone being found, otherwise the procedure is infrared (IR) unsafe.

The second of these problems came to fore in the 1990's [9], when it was realised that there can be stable cones that have two hard particles on opposing edges of the cone and no particles in the middle, e.g. for configurations such as

$$
p_{t 1}>p_{t 2} ; \quad R<D\left(p_{1}, p_{2}\right)<\left(1+p_{t 2} / p_{t 1}\right) R .
$$

In traditional iterative cone algorithms, $p_{1}$ and $p_{2}$ each act as seeds and two stable cones are found, one centred on $p_{1}$, the other centred on $p_{2}$. The third stable cone, centred between $p_{1}$ and $p_{2}$ (and containing them both) is not found. If, however, a soft particle is added between the two hard particles, it too acts as a seed and the third stable cone is then found. The set of stable cones (and final jets) is thus different with and without the soft particle and there is a resulting non-cancellation of divergent real soft production and corresponding virtual contributions, i.e. the algorithm is infrared unsafe.

Infrared unsafety is a serious issue, not just because it makes it impossible to carry out meaningful (finite) perturbative calculations, but also because it breaks the whole relation between the (Born or low-order) partonic structure of the event and the jets that one observes, and it is precisely this relation that a jet algorithm is supposed to codify: it makes no sense for the structure of multi-hundred $\mathrm{GeV}$ jets to change radically just because hadronisation, the underlying event or pileup threw a $1 \mathrm{GeV}$ particle in between them. 
A workaround for the above IR unsafety problem was proposed in [9]: after finding the stable cones that come from the true seed particles, add artificial "midpoint" seeds between pairs of stable cones and search for new stable cones that arise from the midpoint seeds. For configurations with two hard particles, the midpoint fix resolved the IR unsafety issue. It was thus adopted as a recommendation [6] for Run II of the Tevatron and is now coming into use experimentally [10, 11].

Recently, it was observed [1] that in certain triangular three-point configurations there are stable cones that are not identified even by the midpoint procedure. While these can be identified by extended midpoint procedures (e.g. midpoints between triplets of particles) [12, 13], in this article (section 3) we show that there exist yet other 3-particle configurations for which even this fix does not find all stable cones.

Given this history of infrared safety problems being fixed and new ones being found, it seems to us that iterative 1 cone algorithms should be abandoned. Instead we believe that cone jet algorithms should solve the mathematical problem of demonstrably finding all stable cones, i.e. all solutions to eq. (1). This kind of jet algorithm is referred to as an exact seedless cone jet algorithm [6] and has been advocated before in [16]. With an exact seedless algorithm, the addition of one or more soft particles cannot lead to new hard stable cones being found, because all hard stable cones have already been (provably) found. Therefore the algorithm is infrared safe at all orders.

Two proposals exist for approximate implementations of the seedless jet algorithm [6, 17]. They both rely on the event being represented in terms of calorimeter towers, which is far from ideal when considering parton or hadron-level events. Ref. [6] also proposed a procedure for an exact seedless jet algorithm, intended for fixed-order calculations, and implemented for example in the MCFM and NLOJet fixed order (NLO) codes [18, 19, 2 , This method takes a time $\mathcal{O}\left(N 2^{N}\right)$ to find jets among $N$ particles. While perfectly adequate for fixed order calculations $(N \leq 4)$, a recommendation to extend the use of such seedless cone implementations more generally would have little chance of being adopted experimentally: the time to find jets in a single (quiet!) event containing 100 particles would approach $10^{17}$ years.

Given the crucial importance of infrared safety in allowing one to compare theoretical predictions and experimental measurements, and the need for the same algorithm to be used in both, there is a strong motivation for finding a more efficient way of implementing the seedless cone algorithm. Section 4 will show how this can be done, first in the context of a simple one-dimensional example (sec. 4.1), then generalising it to two dimensions ( $y$, $\phi$, sec. 4.2) with an approach that can be made to run in polynomial $\left(N^{2} \ln N\right)$ time. As

\footnotetext{
${ }^{1} \mathrm{~A}$ more appropriate name might be the doubly iterative cone algorithm, since as well as iterating the cones, the cone algorithm's definition has itself seen several iterations since its original introduction by UA1 in 1983 [14, and even since the Snowmass accord [15], the first attempt to formulate a standard, infrared and collinear-safe cone-jet definition, over 15 years ago.

${ }^{2}$ Section 3.4.2 of [6] is the source of some confusion regarding nomenclature, because after discussing both the midpoint and seedless algorithms, it proceeds to show some fixed-order results calculated with the seedless algorithm, but labelled as midpoint. Though both algorithms are IR safe up to the order that was shown, they would not have given identical results.
} 
in recent work on speeding up the $k_{t}$ jet-algorithm [20], the key insights will be obtained by considering the geometrical aspects of the problem. Section 4.3 will discuss aspects of the split-merge procedure.

In section 5 we will study a range of physics and practical properties of the seedless algorithm. Given that the split-merge stage is complex and so yet another potential source of infrared unsafety, we will use Monte Carlo techniques to provide independent evidence for the safety of the algorithm, supplementing a proof given in appendix B. We will examine the speed of our coding of the algorithm and see that it is as fast as publicly available midpoint codes. We will also study the question of the relation between the loworder perturbative characteristics of the algorithm, and its all-order behaviour, notably as concerns the ' $R_{\text {sep }}$ ' issue [21, 1]. Finally we highlight physics contexts where we see similarities and differences between our seedless algorithm and the midpoint algorithm. For inclusive quantities, such as the inclusive jet spectrum, perturbative differences are of the order of a few percent, increasing to $10 \%$ at hadron level owing to reduced sensitivity to the underlying event in the seedless algorithm. For exclusive quantities we see differences of the order of $10-50 \%$, for example for mass spectra in multi-jet events.

\section{Overview of the cone jet-finding algorithm}

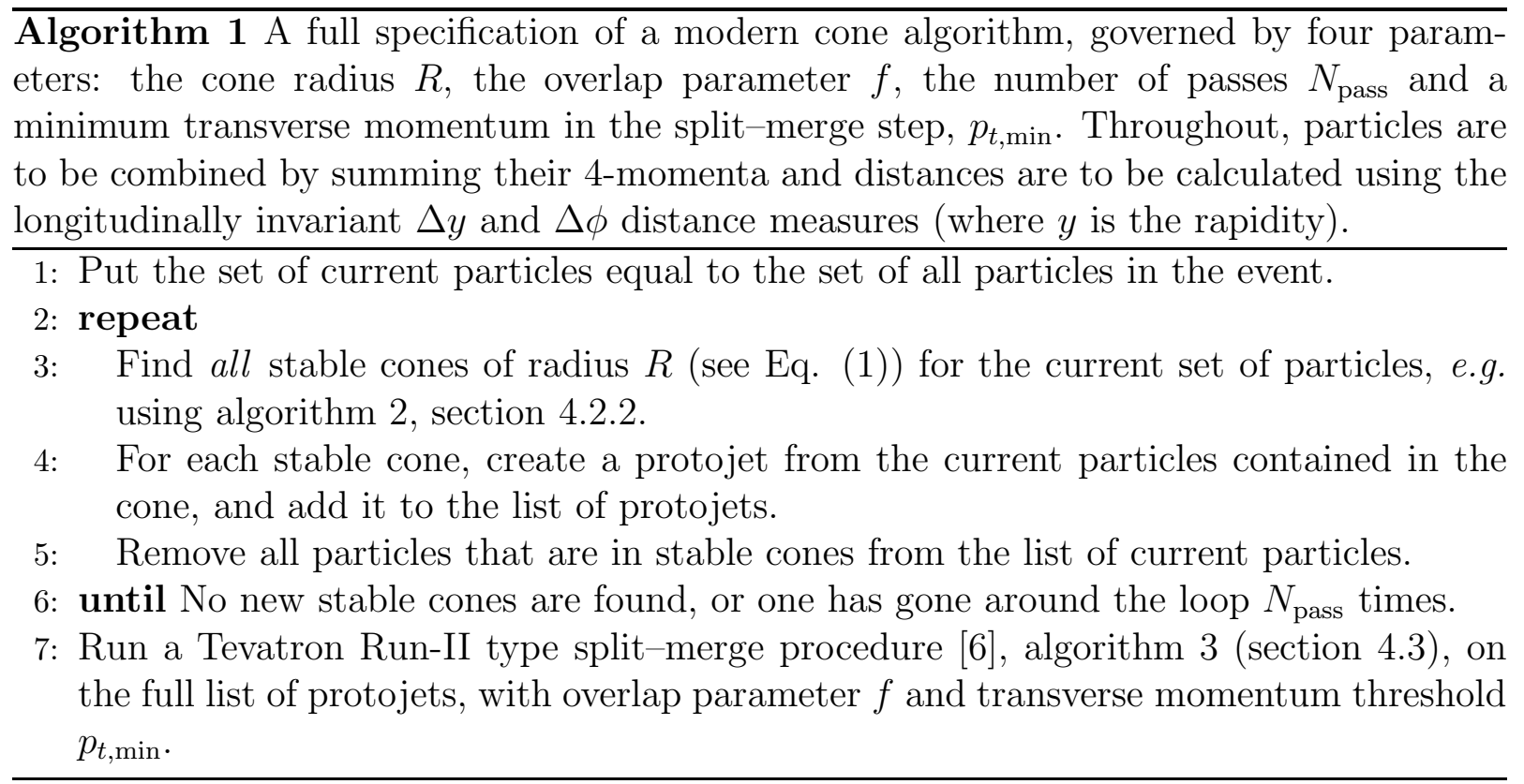

Before entering into technical considerations, we outline the structure of a modern cone jet definition as algorithm 1, largely based on the Tevatron Run-II specification [6]. It is governed by four parameters. The cone radius $R$ and overlap parameter $f$ are standard and appeared in previous cone algorithms. The $N_{\text {pass }}$ variable is new and embodies the suggestion in [1] that one should rerun the stable cone search to eliminate dark towers [21], 


\begin{tabular}{c|c|c|c} 
particle & $p_{t}[\mathrm{GeV}]$ & $y$ & $\phi$ \\
\hline 1 & 400 & 0 & 0 \\
2 & 110 & $0.9 \mathrm{R}$ & 0 \\
3 & 90 & $2.3 \mathrm{R}$ & 0 \\
\hline 4 & 1.1 & $1.5 \mathrm{R}$ & 0
\end{tabular}

Table 1: Particles 1-3 represent a hard configuration. The jets from this hard configuration are modified in the midpoint cone algorithm when one adds the soft particle 4 .

i.e. particles that do not appear in any stable cones (and therefore never appear in jets) during a first pass of the algorithm, even though they can correspond to significant energy deposits. A sensible default is $N_{\text {pass }}=\infty$ since, as formulated, the procedure will in any case stop once further passes find no further stable cones. The $p_{t \text {,min }}$ threshold for the split-merge step is also an addition relative to the Run II procedure, inspired by [12, 7]. It is discussed in section 4.3 together with the rest of the split-merge procedure and may be set to zero to recover the original Run II type behaviour, a sensible default.

The main development of this paper is the specification of how to efficiently carry out step 3 of algorithm [1. In section 3 we will show that the midpoint approximation for finding stable cones fails to find them all, leading to infrared unsafety problems. Section 4 will provide a practical solution. Code corresponding to this algorithm is available publicly under the name of 'Seedless Infrared Safe Cone' (SISCone).

\section{$3 \quad$ IR unsafety in the midpoint algorithm}

Until now, the exact exhaustive identification of all stable cones was considered to be too computationally complex to be feasible for realistic particle multiplicities. Instead, the Tevatron experiments streamline the search for stable cones with the so-called 'midpoint algorithm' 9]. Given a seed, the latter calculates the total momentum of the particles contained within a cone centred on the seed, uses the direction of this momentum as a new seed and iterates until the resulting cone is stable. The initial set of seeds is that of all particles whose transverse momentum is above a seed threshold $s$ (one may take $s=0$ to obtain a collinear-safe algorithm). Then, one adds a new set of seeds given by all midpoints between pairs of stable cones separated by less than $2 R$ and repeats the iterations from these midpoint seeds.

The problem with the midpoint cone algorithm can be seen from the configurations of table 1, represented also in fig. 1. Using particles $1-3$, there exist three stable cones. In a $p_{t}$-scheme recombination procedure (a $p_{t}$ weighted averaging of $y$ and $\phi$ ) they are at $y \simeq\{0.194 R, 1.53 R, 2.3 R\} 3$ Note however that starting from particles $1,2,3$ as seeds, one only iterates to the stable cones at $y \simeq 0.194 R$ and $y=2.3 R$. Using the midpoint between

\footnotetext{
${ }^{3}$ In a more standard $E$-scheme (four-momentum) recombination procedure the exact numbers depend slightly on $R$, but the conclusions are unchanged.
} 

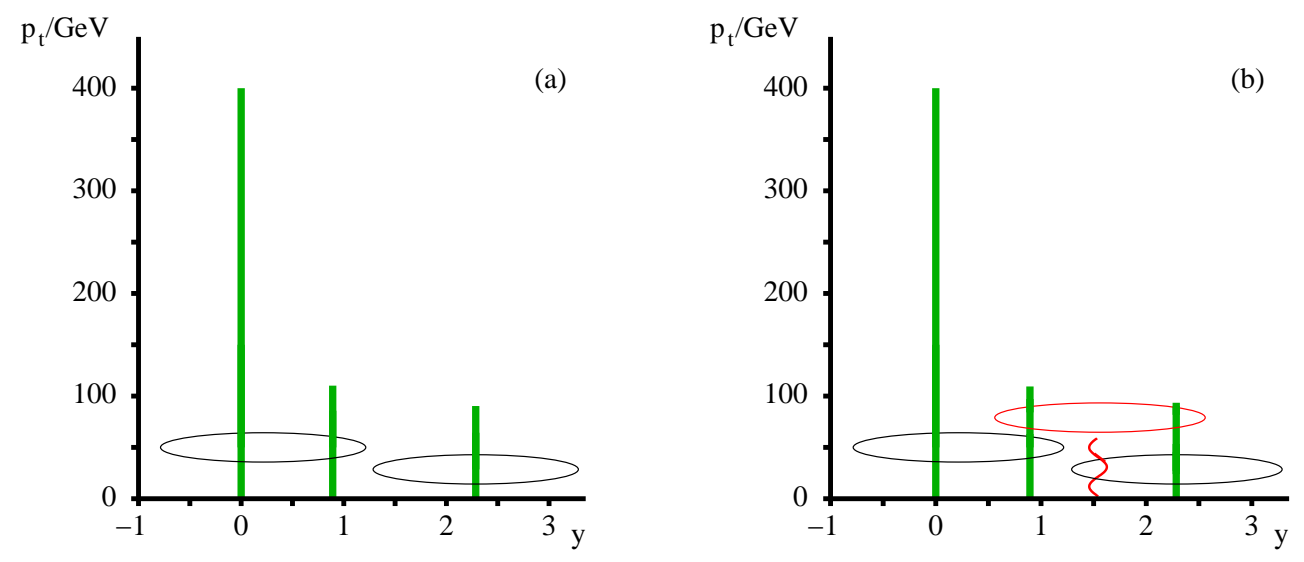

Figure 1: Configuration illustrating one of the IR unsafety problems of the midpoint jet algorithm $(R=1)$; (a) the stable cones (ellipses) found in the midpoint algorithm; (b) with the addition of an arbitrarily soft seed particle (red wavy line) an extra stable cone is found.

these two stable cones, at $y \simeq 1.247 R$, one iterates back to the stable cone at $y \simeq 0.194 R$, therefore the stable cone at $y=1.53 R$ is never found. The result is that particles 1 and 2 are in one jet, and particle 3 in another, fig:1a.

If additionally a soft particle (4) is present to act as a seed near $y=1.53 R$, fig $1 \mathrm{~b}$, then the stable cone there is found from the iterative procedure. In this case we have three overlapping stable cones, with hard-particle content $1+2,2+3$ and 3 . What happens next depends on the precise splitting and merging procedure that is adopted. Using that of [6] then for $f<0.55$ the jets are merged into a single large jet $1+2+3$, otherwise they are split into 1 and $2+3$. Either way the jets are different from those obtained without the extra soft seed particle, meaning that the procedure is infrared unsafe. In contrast, a seedless approach would have found the three stable cones independently of the presence of the soft particle and so would have given identical sets of jets.

The infrared divergence arises for configurations with 3 hard particles in a common neighbourhood plus one soft one (and a further hard electroweak boson or QCD parton to balance momentum). Quantities where it will be seen include the NLO contribution to the heavy-jet mass in $W / Z+2$-jet (or 3 -jet) events, the NNLO contribution to the $W / Z+2$-jet cross section or the 3 -jet cross section, or alternatively at NNNLO in the inclusive jet cross section. The problem might therefore initially seem remote, since the theoretical state of the art is far from calculations of any of these quantities. However one should recall that infrared safety at all orders is a prerequisite if the perturbation series is to make sense at all. If one takes the specific example of the $Z+2$-jet cross section (measured in [10]) then the NNLO divergent piece would be regulated physically by confinement at the non-perturbative scale $\Lambda_{Q C D}$, and would give a contribution of order $\alpha_{E W} \alpha_{s}^{4} \ln p_{t} / \Lambda_{Q C D}$. Since $\alpha_{s}\left(p_{t}\right) \ln p_{t} / \Lambda_{Q C D} \sim 1$, this divergent NNLO contribution will be of the same order as the NLO piece $\alpha_{E W} \alpha_{s}^{3}$. Therefore the NLO calculation has little formal meaning for the midpoint algorithm, since contributions involving yet higher powers of $\alpha_{s}$ 


\begin{tabular}{|l|c|c|}
\hline Observable & 1st miss cones at & Last meaningful order \\
\hline Inclusive jet cross section & NNLO & NLO \\
$W / Z / H+1$ jet cross section & NNLO & NLO \\
3 jet cross section & NLO & LO \\
$W / Z / H+2$ jet cross section & NLO & LO \\
jet masses in 3 jets, $W / Z / H+2$ jets & LO & none \\
\hline
\end{tabular}

Table 2: Summary of the order $\left(\alpha_{s}^{4}\right.$ or $\left.\alpha_{s}^{3} \alpha_{E W}\right)$ at which stable cones are missed in various processes with a midpoint algorithm, and the corresponding last order that can be meaningfully calculated. Infrared unsafety first becomes visible one order beyond that at which one misses stable cones.

will be parametrically as large as the NLO term. 4 The situation for a range of processes is summarised in table 2 ,

\section{An exact seedless cone jet definition}

One way in which one could imagine trying to 'patch' the seed-based iterative cone jetalgorithm to address the above problem would be to use midpoints between all pairs of particles as seeds, as well as midpoints between the initial set of stable cones 5 However it seems unlikely that this would resolve the fundamental problem of being sure that one will systematically find all solutions of eq. (1) for any ensemble of particles.

Instead it is more appropriate to examine exhaustive, non-iterative approaches to the problem, i.e. an exact seedless cone jet algorithm, one that provably finds all stable cones, as advocated already some time ago in [16].

For very low multiplicities $N$, one approach is that suggested in section 3.3.3 of [6] and used in the MCFM [18] and NLOJet [19] next-to-leading order codes. One first identifies all possible subsets of the $N$ particles in the event. For each subset $\mathcal{S}$, one then determines the rapidity $\left(y_{\mathcal{S}}\right)$ and azimuth $\left(\phi_{\mathcal{S}}\right)$ of the total momentum of the subset, $p_{\mathcal{S}}=\sum_{i \in \mathcal{S}} p_{i}$ and then checks whether a cone centred on $y_{\mathcal{S}}, \phi_{\mathcal{S}}$ contains all particles in $\mathcal{S}$ but no other particles. If this is the case then $\mathcal{S}$ corresponds to a stable cone. This procedure guarantees that all solutions to eq. (11) will be found.

In the above procedure there are $\sim 2^{N}$ distinct subsets of particles and establishing whether a given subset corresponds to a stable cone takes time $\mathcal{O}(N)$. Therefore the time to identify all stable cones is $\mathcal{O}\left(N 2^{N}\right)$. For the values of $N(\leq 4)$ relevant in fixedorder calculations, $N 2^{N}$ time is manageable, however as soon as one wishes to consider

\footnotetext{
${ }^{4}$ As concerns the measurement [10], the discussion is complicated by the confusion surrounding the nomenclature of the seedless and midpoint algorithms - while it seems that the measurement was carried out with a true midpoint algorithm, the calculation probably used the 'midpoint' as defined in section 3.4.2 of [6] (cf. footnote 22), which is actually the seedless algorithm, i.e. the measurements and theoretical predictions are based on different algorithms.

${ }^{5}$ This option was actually mentioned in [6] but rejected at the time as impractical.
} 


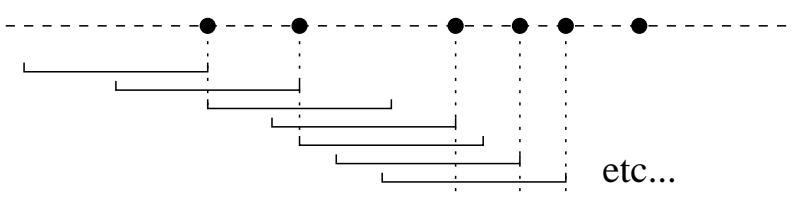

Figure 2: Representation of points on a line and the places where a sliding segment has a change in its set of enclosed points.

parton-shower or hadron-level events, with dozens or hundreds of particles, $N 2^{N}$ time is prohibitive. A solution can only be considered realistic if it is polynomial in $N$, preferably with not too high a power of $N$.

As mentioned in the introduction, approximate procedures for implementing seedless cone jet algorithms have been proposed in the past [6, 17]. These rely on considering the momentum flow into discrete calorimeter towers rather than considering particles. As such they are not entirely suitable for examining the full range event levels, which go from fixedorder (few partons), via parton shower level (many partons) and hadron-level, to detector level which has both tracking and calorimetry information.

\subsection{One-dimensional example}

To understand how one might construct an efficient exact seedless cone jet algorithm, it is helpful to first examine a one-dimensional analogue of the problem. The aim is to identify all solutions to eq. (1), but just for (weighted) points on a line. The equivalent of a cone of radius $R$ is a segment of length $2 R$.

Rather than immediately looking for stable segments one instead looks for all distinct ways in which the segment can enclose a subset of the points on the line. Then for each separate enclosure one calculates its centroid $C$ (weighted with the $p_{t}$ of the particles) and verifies whether the segment centred on $C$ encloses the same set of points as the original enclosure. If it does then $C$ is the centre of a stable segment.

A simple way of finding all distinct segment-enclosures is illustrated in fig,2. First one sorts the points into order on the line. One then places the segment far to the left and slides it so that it goes infinitesimally beyond the leftmost point. This is a first enclosure. Then one slides the segment again until its right edge encounters a new point or the left edge encounters a contained point. Each time either edge encounters a point, the point-content of the segment changes and one has a new distinct enclosure. Establishing the stability of each enclosure is trivial, since one knows how far the segment can move in each direction without changing its point content - so if the centroid is such that the segment remains within these limits, the enclosure corresponds to a stable segment.

The computational complexity of the above procedure, $N \ln N$, is dominated by the need to sort the points initially: there are $\mathcal{O}(N)$ distinct enclosures and, given the sorted list, finding the next point that will enter or leave an edge costs $\mathcal{O}(1)$ time, as does updating the weighted centroid (assuming rounding errors can be neglected), so that the time not associated with the sorting step is $\mathcal{O}(N)$. 

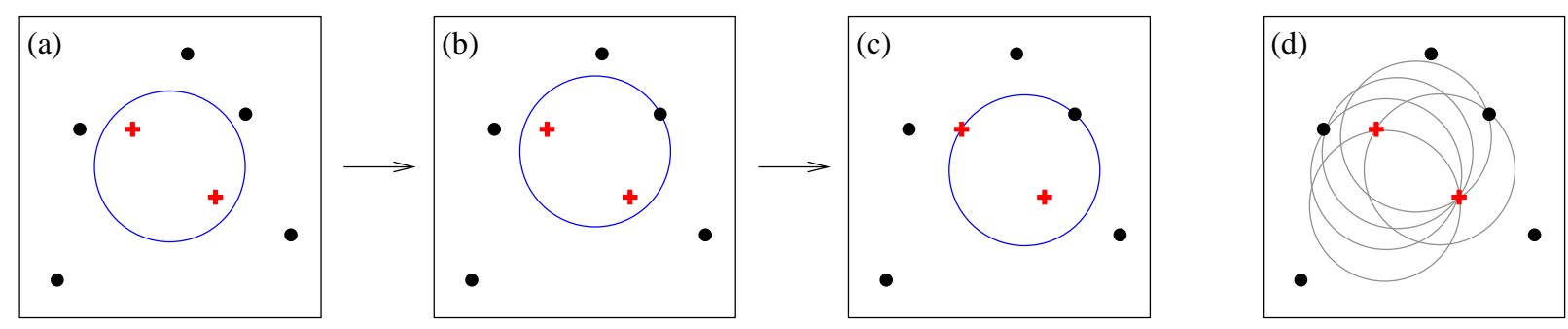

Figure 3: (a) Some initial circular enclosure; (b) moving the circle in a random direction until some enclosed or external point touches the edge of the circle; (c) pivoting the circle around the edge point until a second point touches the edge; (d) all circles defined by pairs of edge points leading to the same circular enclosure.

\subsection{The two-dimensional case}

\subsubsection{General approach}

The solution to the full problem can be seen as a 2-dimensional generalisation of the above procedure 6 The key idea is again that of trying to identify all distinct circular enclosures, which we also call distinct cones (by 'distinct' we mean having a different point content), and testing the stability of each one. In the one-dimensional example there was a single degree of freedom in specifying the position of the segment and all distinct segment enclosures could be obtained by considering all segments with an extremity defined by a point in the set. In 2 dimensions there are two degrees of freedom in specifying the position of a circle, and as we shall see, the solution to finding all distinct circular enclosures will be to examine all circles whose circumference lies on a pair of points from the set.

To see in detail how one reaches this conclusion, it is useful to examine fig. [3. Box (a) shows a circle enclosing two points, the (red) crosses. Suppose, in analogy with fig. 2 that one wishes to slide the circle until its point content changes. One might choose a direction at random and after moving a certain distance, the circle's edge will hit some point in the plane, box (b), signalling that the point content is about to change. In the 1-dimensional case a single point, together with a binary orientation (taking it to be the left or right-hand point) were sufficient to characterise the segment enclosure. However in the 2-dimensional case one may orient the circle in an infinite number of ways. We can therefore pivot the circle around the boundary point. As one does this, at some point a second point will then touch the boundary of the circle, box (c).

The importance of fig. [3 is that it illustrates that for each and every enclosure, one can always move the corresponding circle (without changing the enclosure contents) into a position where two points lie on its boundary 7 Conversely, if one considers each circle

\footnotetext{
${ }^{6}$ We illustrate the planar problem rather than the cylindrical one since for $R<\pi / 2$ the latter is a trivial generalisation of the former.

${ }^{7}$ There are two minor exceptions to this: (a) for any point separated from all others by more than $2 R$, the circle containing it can never have more than that one point on its edge - any such point forms a stable cone of its own; (b) there may be configurations where three or more points lie on the same circle
} 
whose boundary is defined by a pair of points in the set, and considers all four permutations of the edge points being contained or not in the enclosure, then one will have identified all distinct circular enclosures. Note that one given enclosure can be defined by several distinct pairs of particles, which means that when considering the enclosures defined by all pairs of particles, we are likely to find each enclosure more than once, cf. fig. 3 d.

A specific implementation of the above approach to finding the stable cones is given as algorithm 2 below. It runs in expected time $\mathcal{O}(N n \ln n)$ where $N$ is the total number of particles and $n$ is the typical number of particles in a circle of radius $R 8$ The time is dominated by a step that establishes a traversal order for the $\mathcal{O}(N n)$ distinct circular enclosures, much as the one-dimensional $(N \ln N)$ example was dominated by the step that ordered the $\mathcal{O}(N)$ distinct segment enclosures 9 Some aspects of algorithm 2 are rather technical and are explained in the subsubsection that follows. A reader interested principally in the physics of the algorithm may prefer to skip it on a first reading.

\subsubsection{Specific computational strategies}

A key input in evaluating the computational complexity of various algorithms is the knowledge of the number of distinct circular enclosures (or 'distinct cones') and the number of stable cones. These are both estimated in appendix A.1, and are respectively $\mathcal{O}(N n)$ and (expected) $\mathcal{O}(N)$.

Before giving the 2-dimensional analogue of the 1-d algorithm of section 4.1 we examine a simple 'brute force' approach for finding all stable cones. One takes all $\sim N n$ pairs of points within $2 R$ of each other and for each pair identifies the contents of the circle and establishes whether it corresponds to a stable cone, at a cost of $\mathcal{O}(N)$ each time, leading to an overall $N^{2} n$ total cost. This is to be compared to a standard midpoint cone algorithm, whose most expensive step will be the iteration of the expected $\mathcal{O}(N n)$ midpoint seeds, for a total cost also of $N^{2} n$, assuming the average number of iterations from any given seed to be $\mathcal{O}(1) 10$

One can reduce the computational complexity by using some of the ideas from the 1-d example, notably the introduction of an ordering for the boundary points of circles, and the use of the boundary points as sentinels for instability. Specifically, three elements will be required:

i) one needs a way of labelling distinct cones that allows one to test whether two cones are the same at a cost of $\mathcal{O}(1)$;

of radius $R$ (i.e. are cocircular) - given a circle defined by a pair of them, the question of which of the others is in the circle becomes ambiguous and one should explicitly consider all possible combinations of inclusion/exclusion; a specific case of this is when there are collinear momenta (coincident points), which can however be dealt more simply by immediately merging them.

${ }^{8}$ Given a detector that extends to rapidities $y<y_{\max }, n / N \sim \pi R^{2} /\left(4 \pi y_{\max }\right)$, which is considerably smaller than 1 - this motivates us to distinguish $n$ from $N$.

${ }^{9}$ For comparison we note that the complexity of public midpoint algorithm implementations scales as $N^{2} n$.

${ }^{10}$ In both cases one can reduce this to $N n^{2}$ by tiling the plane into squares of edge-length $R$ and restricting the search for the circle contents to tiles in the vicinity of the circle centre. 


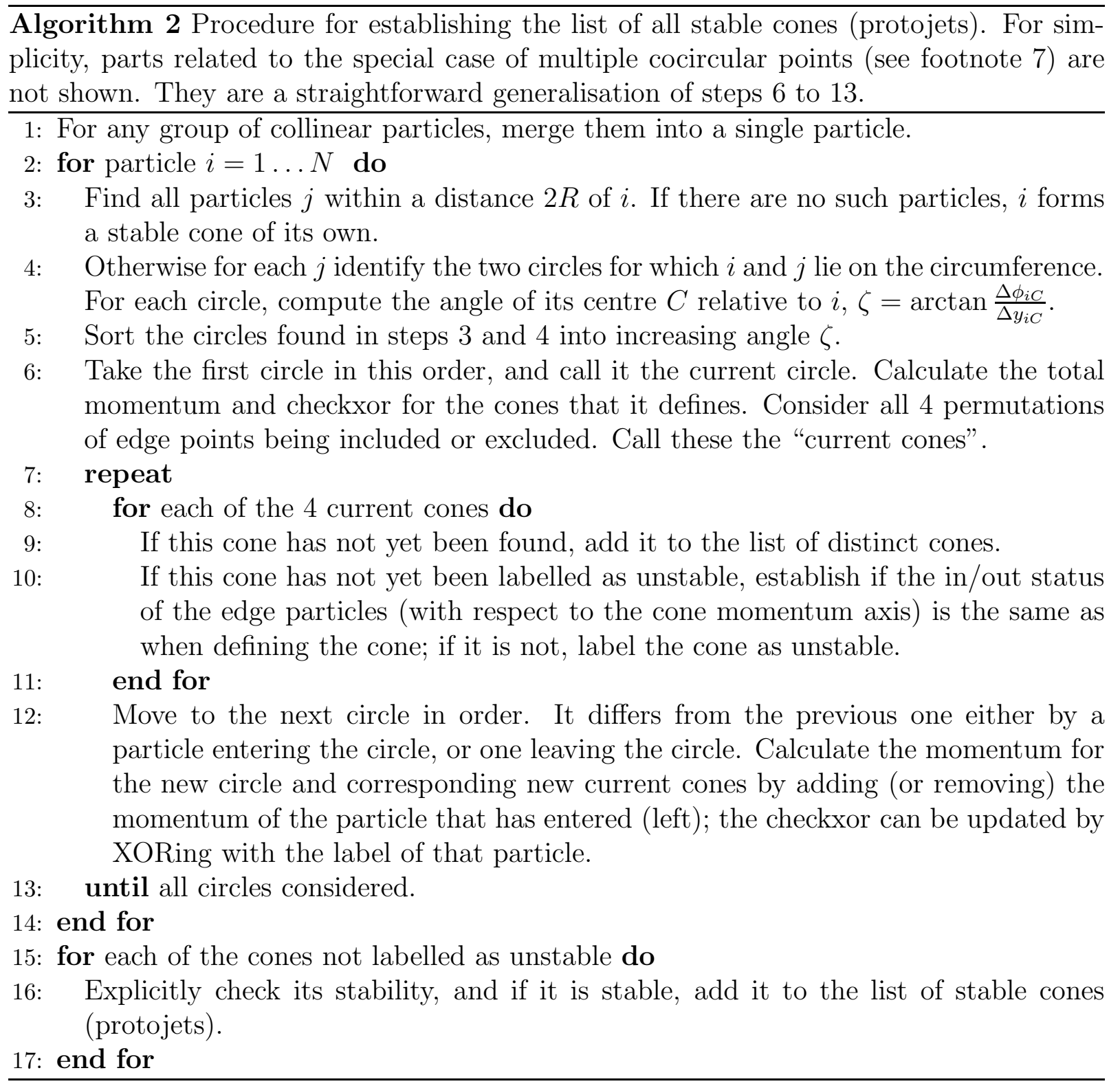


ii) one needs a way of ordering one's examination of cones so that one can construct the cones incrementally, so as not to pay the (at least, see below) $\mathcal{O}(\sqrt{n})$ construction price anew for each cone;

iii) one needs a way limiting the number of cones for which we carry out a full stability test (which also costs at least $\sqrt{n}$ ).

To label cones efficiently, we assign a random $q$-bit integer tag to each particle. Then we define a tag for combinations of particles by taking the logical exclusive-or of all the tags of the individual particles (this is easily constructed incrementally and is sometimes referred to as a checkxor). Then two cones can be compared by examining their tags, rather than by comparing their full list of particles. With such a procedure, there is a risk of two non-identical cones ending up with identical tags ('colliding'), which strictly speaking will make our procedure only 'almost exact'. The probability $p$ of a collision occurring is roughly the square of the number of enclosures divided by the number of distinct tags. Since we have $\mathcal{O}(N n)$ enclosures, this gives $p \sim N^{2} n^{2} / 2^{q}$. By taking $q$ sufficiently large (in a test implementation we have used $q=96$ ) and using a random number generator that guarantees that all bits are decorrelated [22, one can ensure a negligible collision probability 11

Given the ability to efficiently give a distinct label to distinct cones, one can address points ii) and iii) mentioned above by following algorithm 2, Point (ii) is dealt with by steps 2 6, 12 and 13, for each particle $i$, one establishes a traversal order for the circles having $i$ on their edge - the traversal order is such that as one works through the circles, the circle content changes only by one particle at a time, making it easy to update the momentum and checkxor for the circle 12 One maintains a record of all distinct cones in the form of a hash (as a hash function one simply takes $\log _{2} N n$ bits of the tag), so that it only takes $\mathcal{O}(1)$ time to check whether a cone has been found previously.

Rather than explicitly checking the stability of each distinct cone, the algorithm examines whether the multiple edge points that define the cone are appropriately included/excluded in the circle around the cone's momentum axis, step 10, All but a tiny fraction of unstable cones fail this test, so that at the end of step 14 one has a list (of size $\mathcal{O}(N)$ ) of candidate stable cones - at that point one can carry out a full stability test for each of them. This therefore deals with point (iii) mentioned above.

The dominant part of algorithm 2 is the ordering of the circles, step 5, which takes $n \ln n$ time and must be repeated $N$ times. Therefore the overall cost is $N n \ln n$. As well as computing time, a significant issue is the memory use, because one must maintain a list of all distinct cones, of which there are $\mathcal{O}(N n)$. One notes however that standard

\footnotetext{
${ }^{11} \mathrm{~A}$ more refined analysis shows that we need only worry about collisions between the tags of stable cones and other (stable or unstable) cones - since there are $\mathcal{O}(N)$ stable cones, the actual collision probability is more likely to be $\mathcal{O}\left(N n^{2}\right) / 2^{q}$. In practice for $N \sim 10^{4}$ and $n \sim 10^{3}$ (a very highly populated event) and using $q=96$, this gives $p \sim 10^{-18}$. In principle to guarantee an infinitesimal collision probability regardless of $\mathrm{N}, q$ should scale as $\ln N$, however $N$ will in any case be limited by memory use (which scales as $N n$ ) so a fixed $q$ is not unreasonable.

${ }^{12}$ Rounding errors can affect the accuracy of the momentum calculated this way; the impact of this can be minimised by occasionally recomputing the momentum of the circle from scratch.
} 
implementations of the split-merge step of the cone algorithm also require $\mathcal{O}(N n)$ storage, albeit with a smaller coefficient.

It is worth highlighting also an alternative approach, which though slower, $\mathcal{O}\left(\mathrm{Nn}^{3 / 2}\right)$, has lower memory consumption and also avoids the small risk inexactness from the checkxor. It is similar to the brute-force approach, but uses 2-dimensional computational geometry tree structures, such as quad-trees [23] or $k$-d trees [24]. These involve successive sub-divisions of the plane (in quadrants, or pairs of rectangles), similarly to what is done in 1-dimensional binary trees. They make it possible to check the stability of a given circle in $\sqrt{n}$ time (the time is mostly taken by identifying tree cells near the edge of the circle, of which there are $\mathcal{O}(\sqrt{n})$ ), giving an overall cost of $N n^{3 / 2}$. The memory use of this form of approach is $\mathcal{O}(N \sqrt{n})$, simply the space needed to store the stable-cone contents 13

\subsection{The split-merge part of the cone algorithm}

The split-merge part of our cone algorithm is basically that adopted for Run-II of the Tevatron [6]. It is shown in detail as algorithm 3. Since it does not depend on the procedure used to find stable cones, it may largely be kept as is. We do however include the following small modifications:

1. The run II proposal used $E_{t}$ throughout the split-merge procedure. This is not invariant under longitudinal boosts. We replace it with $\tilde{p}_{t}$, a scalar sum of the transverse momenta of the constituents of the protojet. This ensures that the results are both boost-invariant and infrared safe. We note that choosing instead $p_{t}$ (a seemingly natural choice, made for example in the code of [19, 13]) would have led to IR unsafety in purely hadronic events - the question of the variable to be used for the ordering is actually a rather delicate one, and we discuss it in more detail in appendix B.2.

2. We introduce a threshold $p_{t, \text { min }}$ below which protojets are discarded (step 2 of algorithm 3). This parameter is motivated by the discussion in [6] concerning problems associated with an 'excess' of stable cones in seedless algorithms, notably in events with significant pileup. It provides an infrared and collinear safe way of removing the resulting large number of low $p_{t}$ stable cones. By setting it to zero one recovers a behaviour identical to that of the Run-II algorithm (modulo the replacement $E_{t} \rightarrow \tilde{p}_{t}$, above), and we believe that in practice zero is actually a sensible default value. We note that a similar parameter is present in PxCone [12, 7].

\footnotetext{
${ }^{13}$ Though here we are mainly interested in exact approaches, one may also examine the question of the speed of the approximate seedless approach of Volobouev [17. This approach represents the event on a grid and essentially calculates the stability of a cone at each point of the grid using a fast-Fourier transformation (FFT). In principle, for this procedure to be as good as the exact one, the grid should be fine enough to resolve each distinct cone, which implies that it should have $\mathcal{O}(N n)$ points; therefore the FFT will require $\mathcal{O}(N n \ln N n)$ time, which is similar in magnitude to the time that is needed by the exact algorithm. An open question remains that of whether a coarser grid might nevertheless be 'good enough' for many practical applications.
} 


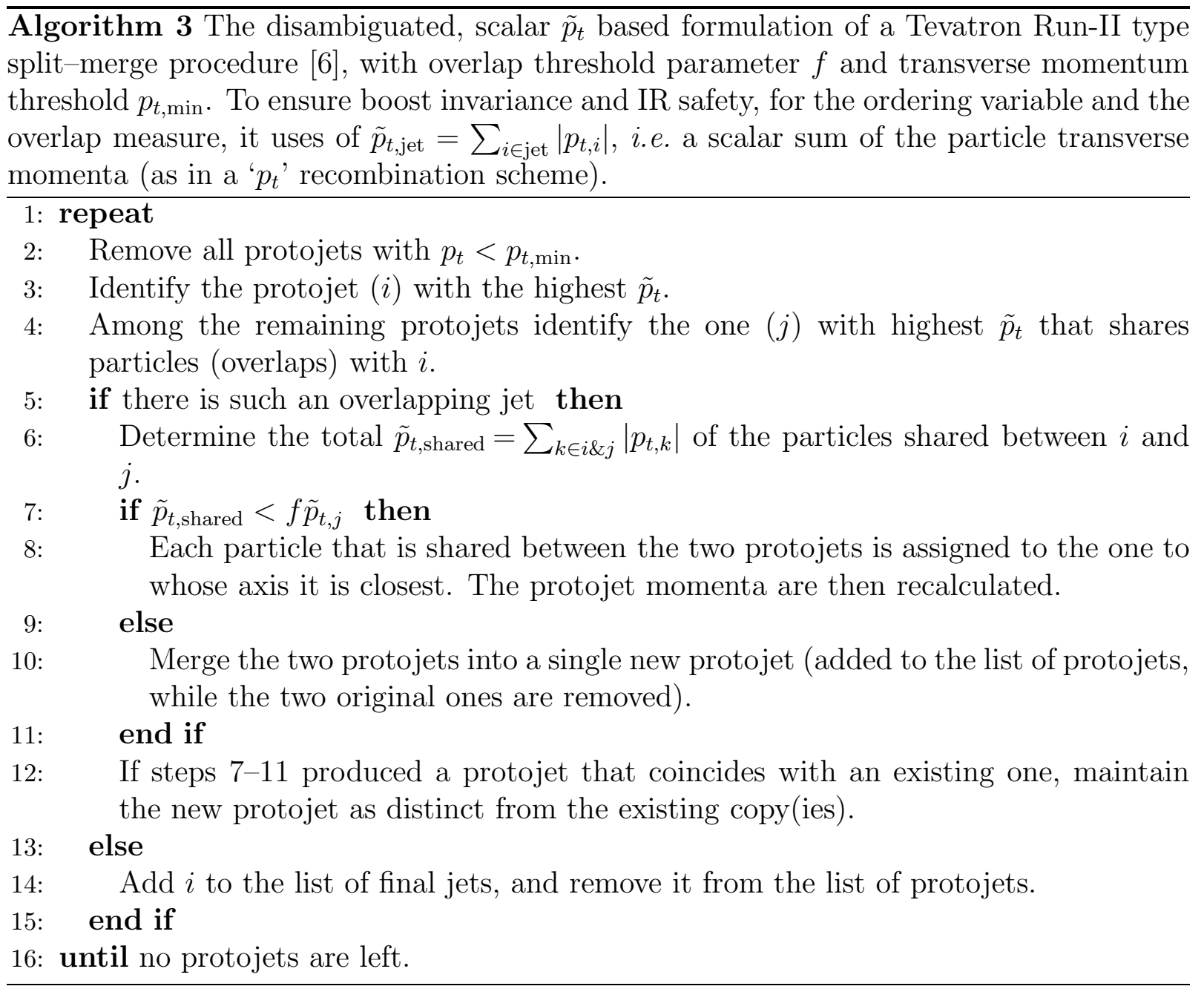

3. After steps 7 11, the same protojet may appear more than once in the list of protojets. For example a protojet may come once from a single original stable cone, and a second time from the splitting of another original stable cone. The original statement of the split-merge procedure [6] did not address this issue, and there is a resulting ambiguity in how to proceed. One option (as is done for example in the seedless cone code of [19]) is to retain only a single copy of any such identical protojets. This however introduces a new source of infrared unsafety: an added soft particle might appear in one copy of the protojet and not the other and the two protojets would then no longer be identical and would not be reduced to a single protojet. This could (and does occasionally, as evidenced in section 5.1) alter the subsequent split-merge sequence. If one instead maintains multiple identical protojets as distinct entities (as is done in the codes of [13, 18]), then the addition of a soft particle does not alter the number of hard protojet entries in the protojet list and the split-merge part of the algorithm remains infrared safe. We therefore choose this second option, and make it explicit as step 12 of algorithm 3 . 
The split-merge procedure is guaranteed to terminate because the number of overlapping pairs of protojets is reduced each time an iteration of the loop finds an overlap. A proof of the infrared safety of this (and the other) parts of our formulation of the cone algorithm is given in appendix $\mathrm{B}$. The computational complexity $\left(\mathcal{O}\left(N^{2}\right)\right)$ of the split-merge procedure is generally smaller than that of the stable-cone search, and so we relegate its discussion to appendix A.2.

Finally, before closing this section, let us return briefly to the top-level of the cone formulation, algorithm 1 and the question of the loop over multiple passes. This loop contains just the stable-cone search, and one might wonder why the split-merge step has not also been included in the loop. First consider $p_{t, \min }=0$ : protojets found in different passes cannot overlap, and the split-merge procedure is such that if a particle is in a protojet then it will always end up in a jet. Therefore it is immaterial whether the splitmerge step is kept inside or outside the loop. The advantage of keeping it outside the loop is that one may rerun the algorithm with multiple overlap values $f$ simply by repeating the split-merge step, without repeating the search for stable cones. For $p_{t, \text { min }} \neq 0$ the positioning of the split-merge step with respect to the $N_{\text {pass }}$ loop would affect the outcome of the algorithm if all particles not found in first-pass jets were to be inserted into the second pass stable-cone search. Our specific formulation constitutes a design choice, which allows one to rerun with different values of $f$ and $p_{t, \text { min }}$ without repeating the stable-cone search.

\section{$5 \quad$ Tests and comparisons}

\subsection{Measures of IR (un)safety}

In section 4 we presented a procedure for finding stable cones that is explicitly IR safe. In appendix $B$ we provide a proof of the IR safety of the rest of the algorithm. The latter is rather technical and not short, and while we have every reason to believe it to be correct, we feel that there is value in supplementing it with complementary evidence for the IR safety of the algorithm. As a byproduct, we will obtain a measure of the IR unsafety of various commonly used formulations of the cone algorithm.

To verify the IR safety of the seedless cone algorithm, we opt for a numerical Monte Carlo approach, in analogy with that used in [25] to test the more involved recursive infrared and collinear safety (a prerequisite for certain kinds of resummation). The test proceeds as follows. One generates a 'hard' event consisting of some number of randomly distributed momenta of the order of some hard scale $p_{t, H}$, and runs the jet algorithm on the hard event. One then generates some soft momenta at a scale $p_{t, S} \ll p_{t, H}$, adds them to the hard event (randomly permuting the order of the momenta) and reruns the jet algorithm. One verifies that the hard jets obtained with and without the soft event are identical. If they are not, the jet algorithm is IR unsafe. For a given hard event one repeats the test

with many different add-on soft events so as to be reasonably sure of identifying most hard events that are IR unsafe. One then repeats the whole procedure for many hard events. 


\begin{tabular}{l|l|l|l} 
Algorithm & Type & IR unsafe & Code \\
\hline JetClu & Seeded, no midpoints & $2 \mathrm{~h}+1 \mathrm{~s}[9]$ & {$[13]$} \\
SearchCone & Seeded, search cone [21], midpoints & $2 \mathrm{~h}+1 \mathrm{~s}[1]$ & {$[13]$} \\
MidPoint & Seeded, midpoints $(2$-way) & $3 \mathrm{~h}+1 \mathrm{~s}[1]$ & {$[13]$} \\
MidPoint-3 & Seeded, midpoints $(2$-way, 3-way) & $3 \mathrm{~h}+1 \mathrm{~s}$ & {$[13]$} \\
PxCone & Seeded, midpoints $(n$-way), non-standard SM & $3 \mathrm{~h}+1 \mathrm{~s}$ & {$[12]$} \\
Seedless [SM- $\left.p_{t}\right]$ & Seedless, SM uses $p_{t}$ & $4 \mathrm{~h}+1 \mathrm{~s}{ }^{a}$ & {$[$ here] } \\
Seedless [SM-MIP] & Seedless, SM merges identical protojets & $4 \mathrm{~h}+1 \mathrm{~s}-$ & {$[$ here] } \\
Seedless [SISCone] & Seedless, SM of algorithm 3 & $n o$ & {$[$ here] }
\end{tabular}

${ }^{a}$ Failures on $4 \mathrm{~h}+1 \mathrm{~s}$ arise only for $R>\pi / 4$; for smaller $R$, failures arise only for higher multiplicities

${ }^{b}$ Failures for $4 \mathrm{~h}+1 \mathrm{~s}$ are extremely rare, but become more common for $5 \mathrm{~h}+1 \mathrm{~s}$ and beyond

Table 3: Summary of the various cone jet algorithms and the code used for tests here; $\mathrm{SM}$ stands for "split-merge"; $N \mathrm{~h}+M \mathrm{~s}$ indicates that infrared unsafety is revealed with configurations consisting of $N$ hard particles and $M$ soft ones, not counting an additional hard, potentially non-QCD, particle to conserve momentum. All codes have been used in the form of plugins to FastJet (v2.1) [20].

The hard events are produced as follows: we choose a linearly distributed random number of momenta (between 2 and 10) and for each one generate a random $p_{t}$ (linearly distributed, $2^{-24} p_{t, H} \leq p_{t} \leq p_{t, H}$, with $p_{t, H}=1000 \mathrm{GeV}$ ), a random rapidity (linearly distributed in $-1.5<y<1.5)$ and a random $\phi$. For each hard event we also choose random parameters for the jet algorithm, so as to cover the jet-algorithm parameter space $(0.3<R<1.57,0.25<f<0.95$, linearly distributed, the upper limit on $R$ being motivated by the requirement that $R<\pi / 2$; the $p_{t, \text { min }}$ on protojets is set to 0 and the number of passes is set to 1 ). For each add-on soft event we generate between 1 and 5 soft momenta, distributed as the hard ones, but with the soft scale $p_{t, S}=10^{-100} \mathrm{GeV}$ replacing $p_{t, H}$.

We note that the hard events generated as above do not conserve momentum - they are analogous to events with a missing energy component or with identified photons or leptons that are not given as inputs to the jet clustering. For the safety studies on the full SISCone algorithm, we therefore also generate a set of hard events which do have momentum conservation, analogous to purely hadronic events.

To validate our approach to testing IR safety, we apply it to a range of cone jet algorithms, listed in table 3 , including the many variants that are IR unsafe. In PxCone the cut on protojets is set to $1 \mathrm{GeV}$ and in the SearchCone algorithm the search cone radius is set to $R / 2$.

The fraction of hard events failing the safety test is shown in fig. 4 for each of the jet algorithms 14 All jet algorithms that are known to be IR unsafe do indeed fail the tests.

\footnotetext{
${ }^{14}$ The results are based on 80 trial soft add-on events for each hard event and should differ by no more than a few percent (relative) from a full determination of the IR safety for each hard event (which would be obtained in the limit of an infinite number of trial soft add-on events for each hard event). For SISCone we only use 20 soft add-on events, so as to make it possible to probe a larger number of hard configurations.
} 


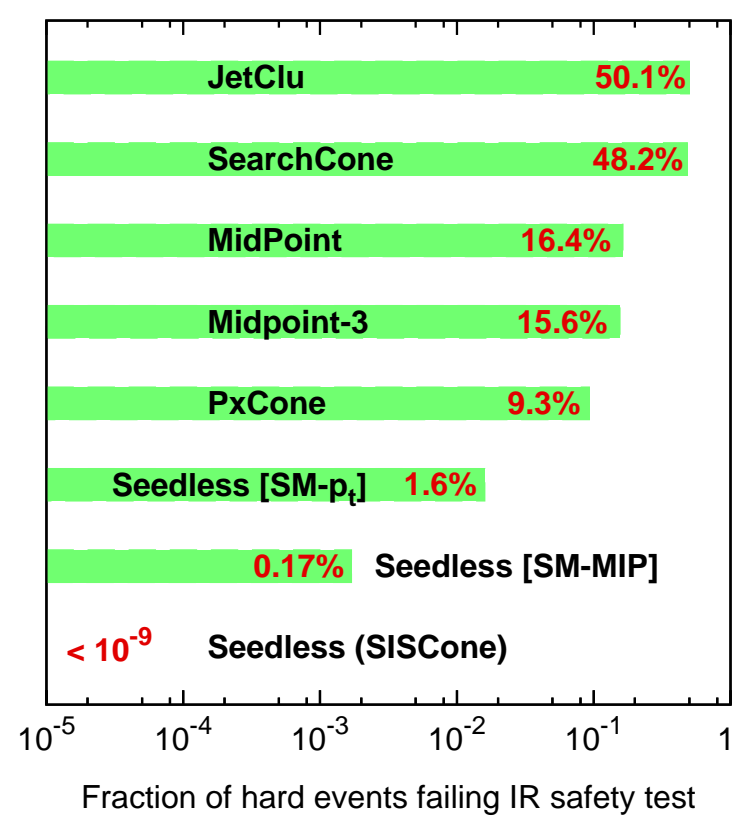

Figure 4: Failure rates for the IR safety tests. The algorithms are as detailed in table 3 , Seeded algorithms have been used with a zero seed threshold. The events used do not conserve momentum (i.e. have a missing energy component), except for the seedless SM- $p_{t}$ case (where all events conserve momentum, to highlight the issue that arises in that case) and for SISCone (where we use a mix of momentum conserving and non-conserving events so as to fully test the algorithm). Further details are given in the text

One should be aware that the absolute failure rates depend to some extent on the way we generated the hard events, and so are to be interpreted with caution. Having said that, our hard events have a complexity similar to the Born-level (lowest-order parton-level) of events that will be studied at LHC, for example in the various decay channels of $t \bar{t} H$ production, and so both the order of magnitudes of the failure rates and their relative sizes should be meaningful.

Algorithms that fail on ' $2 \mathrm{~h}+1 \mathrm{~s}$ ' events have larger failure rates than those that fail on ' $3 \mathrm{~h}+1 \mathrm{~s}$ ' events, as would be expected - they are 'more' infrared unsafe. One notes the significant failure rates for the midpoint algorithms, $\sim 16 \%$, and the fact that adding 3-way midpoints (i.e. between triplets of stable cones) has almost no effect on the failure rate, indicating that triangular configurations identified as IR unsafe in [1] are much less important than others such as that discussed in section 3. PxCone's smaller failure rate seems to be due not to its multi-way midpoints, but rather to its specific split-merge procedure which leads to fewer final jets (so that one is less sensitive to missing stable cones).

Seedless algorithms with problematic split-merge procedures lead to small failure rates (restricting one's attention to small values of $R$, these values are further reduced). One might be tempted to argue that such small rates of IR safety failure are unlikely to have a physical impact and can therefore be ignored. However there is always a risk of some 
specific study being unusually sensitive to these configurations, and in any case our aim here is to provide an algorithm whose IR safety is exact, not just approximate.

Finally, with a 'good' split-merge procedure, that given as algorithm 3, none of the over $5 \times 10^{9}$ hard events tested (a mix both with and without momentum conservation) failed the IR safety test. For completeness, we have carried out limited tests also for $N_{\text {pass }}=\infty$ and with a $p_{t, \text { min }}$ on protojets of $100 \mathrm{GeV}$, and have additionally performed tests with a larger range of rapidities $(|y|<3)$, collinearly-split momenta, cocircular configurations, three scales instead of two scales and again found no failures. These tests together with the proof given in appendix B give us a good degree of confidence that the algorithm truly is infrared safe, hence justifying its name.

\subsection{Speed}

As can be gathered from the discussion in [6], reasonable speed is an essential requirement if a new variant of cone jet algorithm is to be adopted. To determine the speed of various cone jet algorithms, we use the same set of events taken for testing the FastJet formulation of the $k_{t}$ jet algorithm in [20] - these consist of a single Pythia [26] dijet event (with $\left.p_{t, \text { jets }} \simeq 50 \mathrm{GeV}\right)$ to which we add varying numbers of simulated minimum bias events so as to vary the multiplicity $N$. Thus the event structure should mimic that of LHC events with pileup.

Figure 5 shows the time needed to find jets in one event as a function of $N$. Among the seeded jet algorithms we consider only codes that include midpoint seeds. For the (CDF) midpoint code [13], written in $\mathrm{C++}$, there is an option of using only particles above a threshold $s$ as seeds and we consider both the common (though collinear unsafe) choice $s=1 \mathrm{GeV}$ and the (collinear safe but IR unsafe) $s=0 \mathrm{GeV}$. The PxCone code [12], written in Fortran 77, has no seed threshold.

Our seedless code, SISCone, is comparable in speed to the fastest of the seeded codes, the CDF midpoint code with a seed threshold $s=1 \mathrm{GeV}$, and is considerably faster than the codes without a seed threshold (not to mention existing exact seedless codes which take $\sim 1$ s to find jets among 20 particles and scale as $N 2^{N}$ ). Its run time also increases more slowly with $N$ than that of the seeded codes, roughly in agreement with the expectation

of SISCone going as $N n \ln n$ (with a large coefficient) while the others go as $N^{2} n$. The midpoint code with $s=1 \mathrm{GeV}$ has a more complex $N$-dependence presumably because we have run the timing on a single set of momenta, and the proportionality between the number of seeds and $N$ fluctuates and depends on the event structure.

For comparison purposes we have also included the timings for the FastJet (v2) $k_{t}$ implementation, which for these values of $N$ uses a strategy that involves a combination of $N \ln N$ and $N n$ dependencies. Timings for the FastJet implementation of the Aachen/Cambridge algorithm are similar to those for the $k_{t}$ algorithm. 


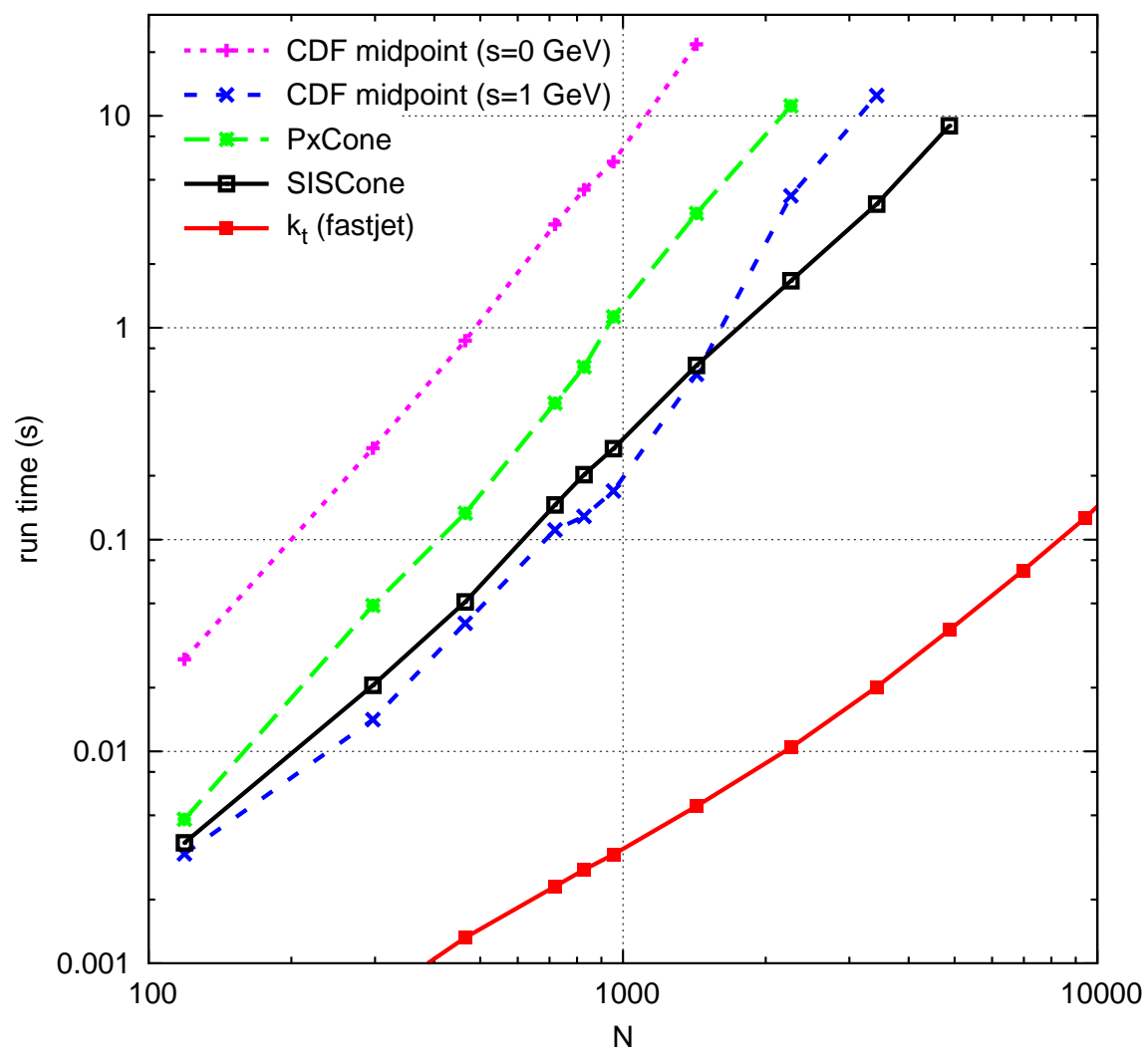

Figure 5: Time to cluster $N$ particles, as a function of $N$, for various algorithms, with $R=0.7$ and $f=0.5$, on a $3.4 \mathrm{GHz}$ Pentium $\AA$ IV processor. For the CDF midpoint algorithm, $s$ is the threshold transverse momentum above which particles are used as seeds.

\section{$5.3 \quad R_{\text {sep }}$ : an inexistent problem}

Suppose we have two partons separated by $\Delta R$ and with transverse momenta $p_{t 1}$ and $p_{t 2}$ $\left(p_{t 1}>p_{t 2}\right)$. Both partons end up in the same jet if the cone containing both is stable, i.e. if

$$
\frac{\Delta R}{R}<1+z, \quad z=\frac{p_{t 2}}{p_{t 1}},
$$

where the result is exact for small $R$ or with $p_{t}$-scheme recombination. Equivalently one can write the probability for two partons to be clustered into a single jet as

$$
P_{2 \rightarrow 1}(\Delta R, z)=\Theta\left(1+z-\frac{\Delta R}{R}\right) .
$$

The limit on $\Delta R / R$ ranges from 1 for $z=0$ to 2 for $z=1$. This $z$-dependent limit is the main low-order perturbative difference between the cone algorithm and inclusive versions of sequential recombination ones like the $k_{t}$ or Cambridge/Aachen algorithms, since the latter merge two partons into a single jet for $\Delta R / R<1$, independently of their energies. 


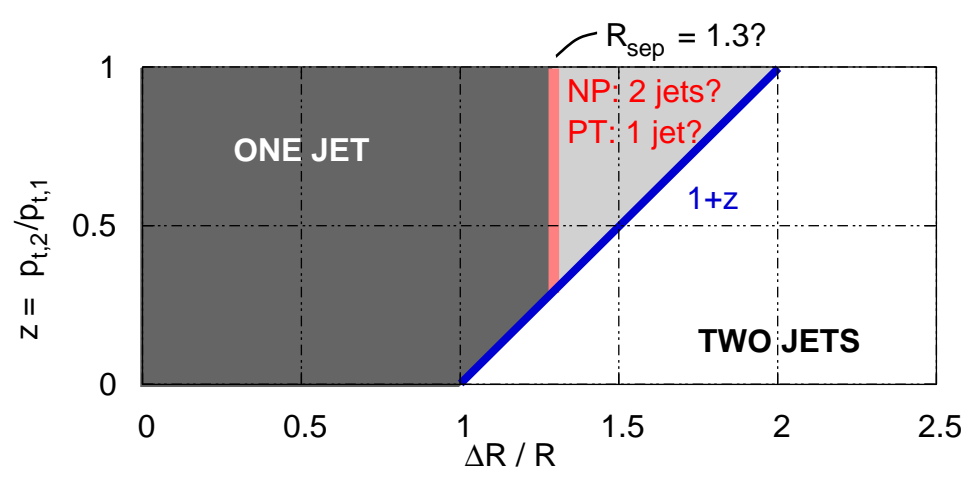

Figure 6: Schematic representation of the phase space region in which two partons will end up in a single cone jet versus two jets, at the 2-parton level (PT) and, according to the $R_{\text {sep }}$ statement, after showering and hadronisation (NP).

A statement regularly made about cone algorithms (see for example [21, 1, 27]) is that parton showering and hadronisation reduce the stability of the cone containing the 'original' two partons, leading to a modified 'practical' condition for two partons to end up in a single jet,

$$
\frac{\Delta R}{R}<\min \left(R_{\mathrm{sep}}, 1+z\right)
$$

or equivalently,

$$
P_{2 \rightarrow 1}(\Delta R, z)=\Theta\left(1+z-\frac{\Delta R}{R}\right) \Theta\left(R_{\text {sep }}-\frac{\Delta R}{R}\right)
$$

with $R_{\text {sep }} \simeq 1.3$ [28, 29] 15 This situation is often represented as in figure 6, which depicts the $\Delta R, z$ plane, and shows the regions in which two partons are merged into one jet or resolved as two jets. The boundary $\Delta R=1+z$ corresponds to eq. (3), while the alternative boundary at $\Delta R=R_{\text {sep }}$ is eq. (5) .

So large a difference between the low-order partonic expectation and hadron-level results would be quite a worrying feature for a jet algorithm - after all, the main purpose of a jet algorithm is to give as close a relation as possible between the first couple of orders of perturbation theory and hadron level 16

The evidence for the existence of eq. (6) with $R_{\mathrm{sep}}=1.3$ seems largely to be based [28, 29 on merging two events (satisfying some cut on the jet $p_{t}$ 's), running the jet-algorithm on the merged event, and examining at what distance particles from the two events end up in the same jet. This approach indicated that particles were indeed less likely to end

\footnotetext{
${ }^{15}$ The name $R_{\text {sep }}$ was originally introduced $[30$, in the context of NLO calculations of hadron-collider jet-spectra, but with a different meaning - there it was intended as a free parameter to model the lack of knowledge about the details of the definition of the cone jet algorithm used experimentally. This is rather different from the current use as a parameter intended to model our inability to directly calculate the impact of higher-order and non-perturbative dynamics of QCD in cone algorithms.

${ }^{16}$ The apparent lack of correspondence is considered sufficiently severe that in some publications (e.g. [11]) the NLO calculation is modified by hand to compensate for this.
} 


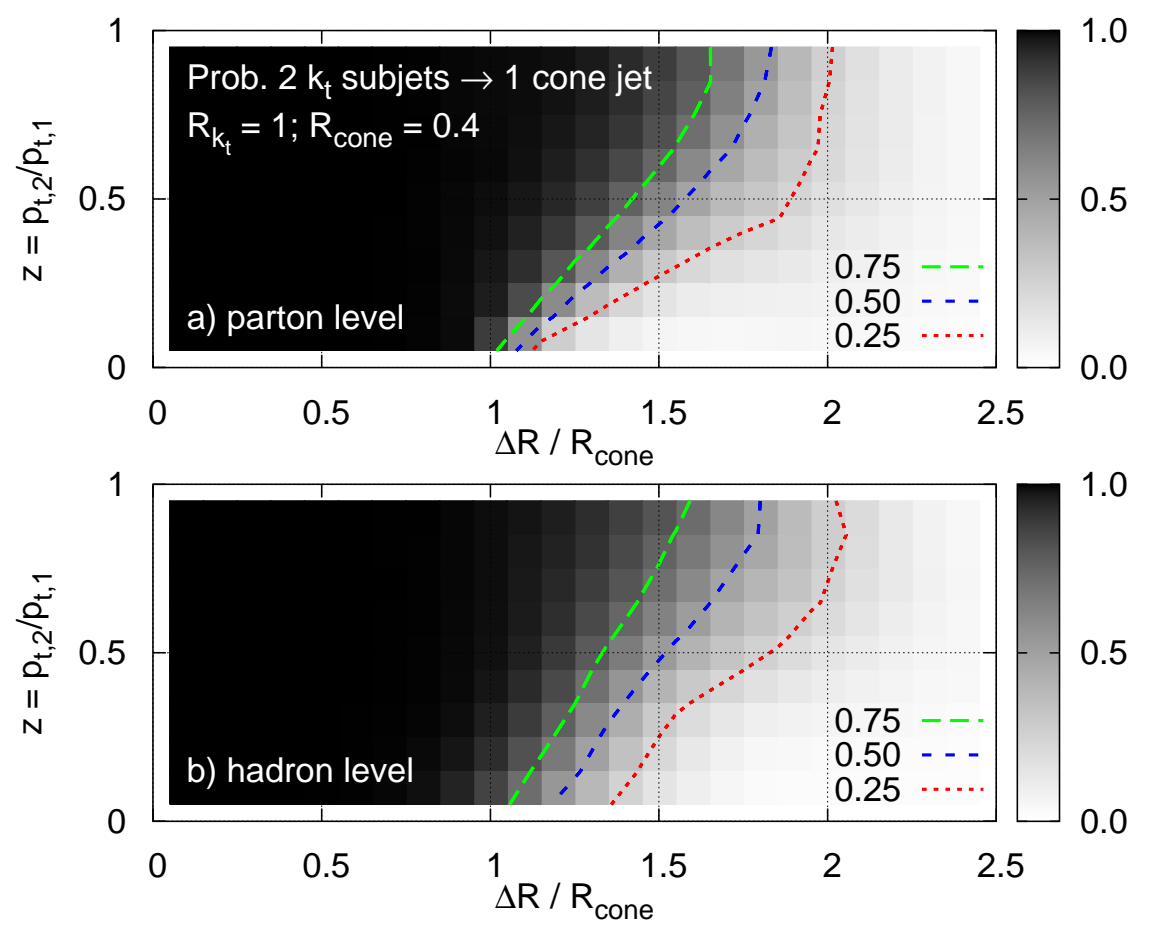

Figure 7: The probability $P_{2 \rightarrow 1}(\Delta R, z)$ for two $k_{t}$-algorithm subjets to correspond to a single cone jet, as a function of $p_{t 1} / p_{t 2}$ and $\Delta R$ for the two $k_{t}$ subjets. Events have been generated with Herwig [31] (hadron-level includes the underlying event) and the results are based on studying all $k_{t}$ jets with $p_{t}>50 \mathrm{GeV}$ and $|y|<1$. Further details are to be found in the text.

up in the same jet if they were more than $1.3 R$ apart, however the result is an average over a range of $z$ values making it hard to see whether eq. (6) is truly representative of the underlying physics 17

To address the question in more depth we adopt the following strategy. Rather than combining different events, we use one event at a time, but with two different jet algorithms. On one hand we run SISCone with a fairly small value of $R, R_{\text {cone }}=0.4$. Simultaneously we run inclusive $k_{t}$ jet-clustering [2] on the event, using a relatively large $R\left(R_{k_{t}}=1.0\right)$, and identify any hard $k_{t}$-jets. For each hard $k_{t}$ jet we undo its last clustering step so as to obtain two subjets, $S_{1}$ and $S_{2}$ - these are taken to be the analogues of the two partons. We then examine whether there is a cone jet that contains more than half of the $p_{t}$ of each of $S_{1}$ and $S_{2}$. If there is, the conclusion is that the two $k_{t}$ subjets have ended up (dominantly) in a single cone jet.

The procedure is repeated for many events, and one then examines the probability, $P_{2 \rightarrow 1}(\Delta R, z)$, of the two $k_{t}$ subjets being identified with a single cone jet, as a function of the distance $\Delta R$ between the two subjets, $S_{1}$ and $S_{2}$, and the ratio $z$ of their $p_{t}$ 's. The

\footnotetext{
${ }^{17} \mathrm{~A}$ preliminary version of [27] showed more differential results; these, however, seem not to be in the definitive version.
} 
results are shown in fig. 7 both at parton-shower level and at hadron level, as simulated with Herwig [31]. The middle contour corresponds to a probability of $1 / 2$. At partonshower level this contour coincides remarkably well with the boundary defined by eq. (3), up to $\Delta R / R=1.7$. It is definitely not compatible with eq. (5) with $R_{\text {sep }}=1.3$. Beyond $\Delta R / R=1.7$ the contour bends a little and one might consider interpreting this as an $R_{\text {sep }} \simeq 1.818$ However, in that region the transition between $P=1$ and $P=0$ is broad, and to within the width of the transition, there remains good agreement with eq. (3) - it seems more natural therefore to interpret the small deviation from eq. (3) as a Sudakovshoulder type structure [32, which broadens and shifts the $\Theta$-function of eq. (44), as would happen with almost any discontinuity in a leading-order QCD distribution.

Once one includes hadronisation effects in the study, fig. $7 \mathrm{~b}$, one finds that the transition region broadens further, as is to be expected. Now the $P=1 / 2$ contour shifts away slightly from the $1+z$ result at small $z$ as well. However, once again this shift is modest, and of similar size as the breadth of the transition region.

To verify the robustness of the above results we have examined other related indicators. One of them is the probability, $P_{2 \rightarrow 2}$ of finding two cone jets, each containing more than half of the transverse momentum of just one of the $k_{t}$ subjets. At two-parton level, one expects $P_{1 \rightarrow 2}+P_{2 \rightarrow 2}=1$. Deviation from this would indicate that our procedure for matching cone jets to $k_{t}$ jets is misbehaving. We find that the relation holds to within around $15 \%$ over most of the region, deviating by at most $\sim 25 \%$ in a small corner of phase space $\Delta R / R \simeq 1.5, z \simeq 0.2$. Another test is to examine the fraction $F_{2}$ of the softer $S_{2}$ 's transverse momentum that is found in the cone that overlaps dominantly with $S_{1}$. At twoparton level this should be equal to $P_{2 \rightarrow 1}$, but this would not be the case after showering if there were underlying problems with our matching procedure. We find however that $F_{2}$ does agree well with $P_{2 \rightarrow 1}$. These, together with yet further tests, lead to us to believe that conclusions drawn from fig. 7 are robust. Finally, while these results have been obtained within a Monte Carlo simulation, Herwig, a similar study could equally be well carried experimentally on real events.

So, in contrast to statements that are often made about the cone jet algorithm, the perturbative picture of when two partons will recombine, given by eq. (44), seems to be a relatively good indicator of what happens even after perturbative radiation and hadronisation. In particular the evidence that we have presented strongly disfavours the $R_{\mathrm{sep}}$-based modification, eq. (6). This is a welcome finding, and should help provide a firmer basis for cone-based phenomenology.

\subsection{Physics impact of seedless v. midpoint cone}

In this section, we discuss the impact on physical measurement of switching from a midpoint type algorithm to a seedless IR-safe one such as SISCone. We study two physical observables, the inclusive jet spectrum and the jet mass spectrum in 3-jet events. The

\footnotetext{
${ }^{18}$ Such a value has been mentioned to us independently by M. Wobisch in the context of unpublished studies of jet shapes for the SearchCone algorithm [21].
} 
spectra have been obtained by generating events with a Monte-Carlo either at fixed order in perturbation theory (NLOJet [19]) or with parton showering and hadronisation (Pythia [26]), and by performing the jet analysis on each event using three different algorithms (each with $R=0.7$ and $f=0.5$, and additionally in the case of SISCone, $N_{\text {pass }}=1$ and $\left.p_{t, \min }=0\right)$ :

1. SISCone: the seedless, IR-safe definition described in algorithms 13 ,

2. midpoint(0): the midpoint algorithm using all particles as seeds;

3. midpoint(1): the midpoint algorithm using as seeds all particles above a threshold of $1 \mathrm{GeV}$.

We have used a version of the CDF implementation of the midpoint algorithm modified to have the split-merge step based on $\tilde{p}_{t}$ rather than $p_{t}$ (so that it corresponds to algorithm4.3 with $\left.p_{t, \text { min }}=0\right)$. The motivation for this is that we are mainly interested in the physics impact of having midpoint versus all stable cones, and the comparison is simplest if the subsequent split-merge procedure is identical in both cases 19

We shall first present the results obtained for the inclusive jet spectrum and then discuss the jet mass spectrum in 3-jet events. Most studies carried out in this section have used kinematics corresponding to the Tevatron Run II, i.e. a centre-of-mass energy $\sqrt{s}=1.96$ $\mathrm{TeV}$, and usually, for simplicity we have chosen not to impose any cuts in rapidity.

\subsubsection{Inclusive jet spectrum}

As discussed in section 3, the differences between the midpoint algorithm and SISCone are expected to start when we have 3 particles in a common neighbourhood plus one to balance momentum. For pure QCD processes this corresponds to $2 \rightarrow 4$ diagrams, $\mathcal{O}\left(\alpha_{s}^{4}\right)$. This is NNLO for the inclusive spectrum. Though a NNLO calculation of the inclusive spectrum is beyond today's technology (for recent progress, see [33]), we can easily calculate the $\mathcal{O}\left(\alpha_{s}^{4}\right)$ difference between midpoint and SISCone, using just tree-level $2 \rightarrow 4$ diagrams, since the difference between the algorithms is zero at orders $\alpha_{s}^{2}$ and $\alpha_{s}^{3}$, i.e. we can neglect two-loop $2 \rightarrow 2$ diagrams and one-loop $2 \rightarrow 3$ diagrams. The significance of the difference can be understood by comparing to the leading order spectrum, which is identical for the two algorithms.

Figure 8 shows the resulting spectra: the upper plot gives the leading order inclusive spectrum together with the difference between SISCone and midpoint $(0)$ at $\mathcal{O}\left(\alpha_{s}^{4}\right)$. The lower plot shows the relative difference. One sees that the use of the IR-safe seedless cone algorithm introduces modest corrections, of order 1-2\%, in the inclusive jet spectrum. This order of magnitude is roughly what one would expect, since the differences only appear at

\footnotetext{
${ }^{19}$ We could also have compared SISCone with a midpoint algorithm using $p_{t}$ in the split-merge (a common default); the figures we show below would have stayed unchanged at the $1 \%$ level for the inclusive spectrum, while for the jet masses the effects range between a few percent at moderate masses and $10-20 \%$ in the high-mass tail.
} 

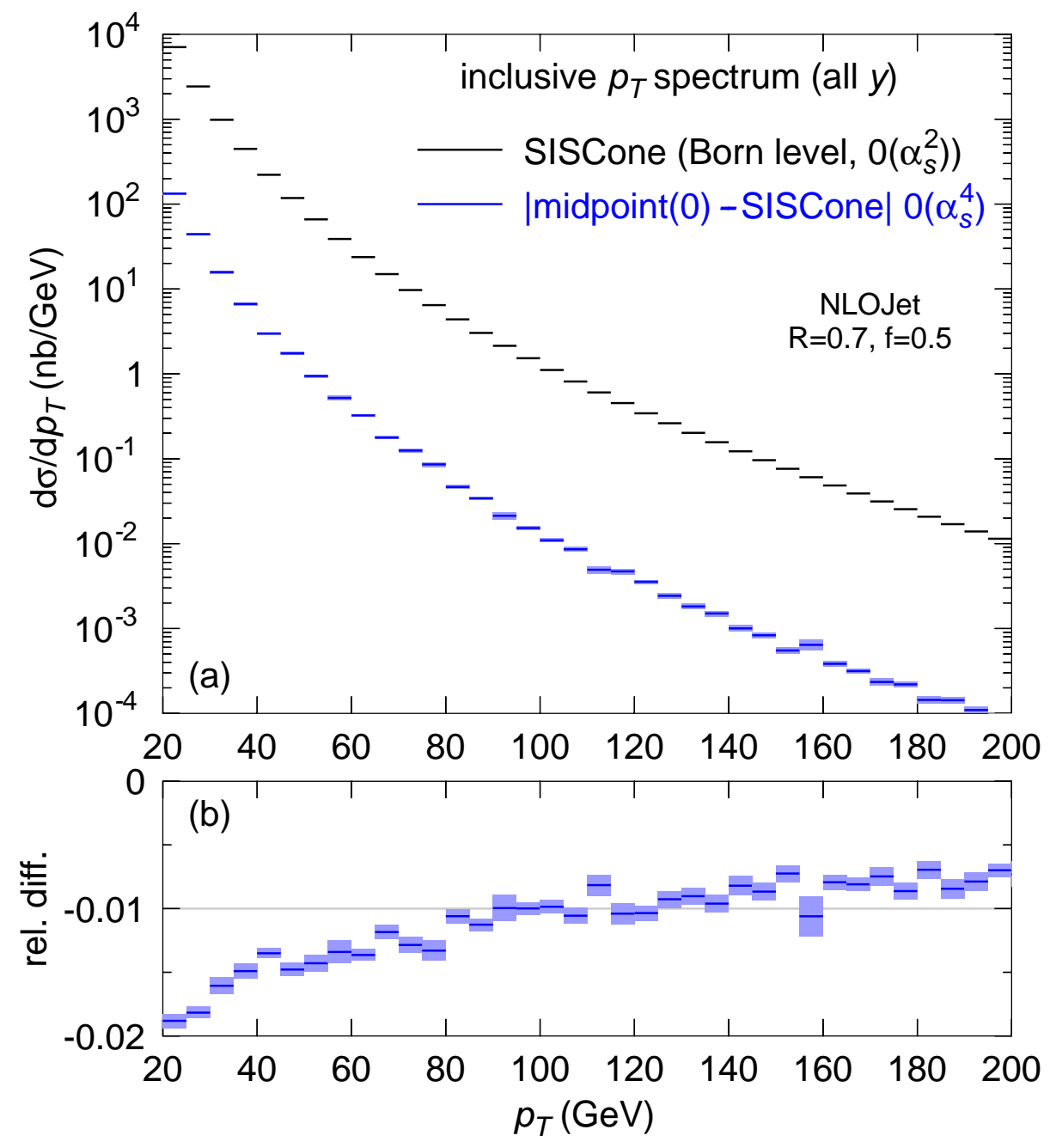

Figure 8: (a) Inclusive jet spectrum: the upper curve gives the leading-order $\left(\mathcal{O}\left(\alpha_{s}^{2}\right)\right)$ spectrum, while the lower (blue) curve gives the difference between the SISCone and midpoint(0) algorithm, obtained from the $\mathcal{O}\left(\alpha_{s}^{4}\right)$ tree-level amplitude; (b) the relative difference. 

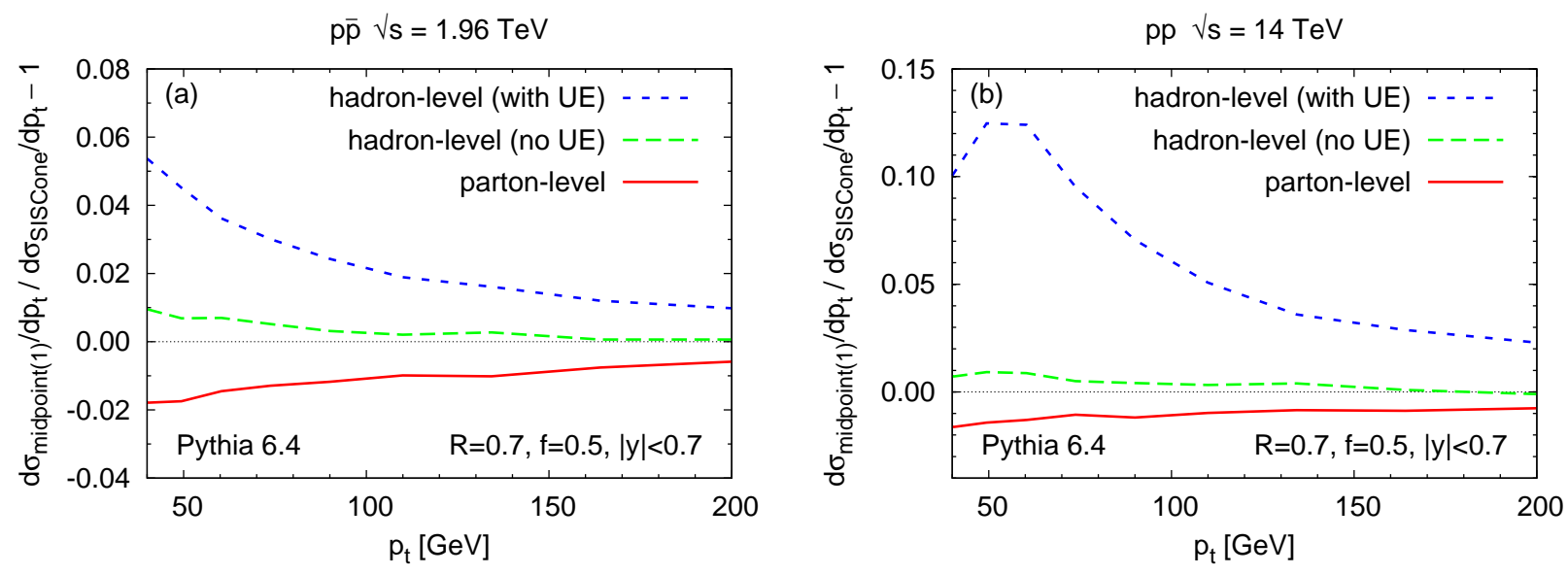

Figure 9: Relative difference between the inclusive jet spectra for midpoint(1) and SISCone, obtained from Pythia at parton level, hadron level without underlying event (UE) contributions, and hadron level with UE. Shown (a) for Tevatron collisions and (b) for LHC collisions.

relative order $\alpha_{s}^{2}$. As we will see below, larger differences will appear when one examines more exclusive quantities.

In addition, we have used Herwig and Pythia to investigate the differences between midpoint(1) and SISCone with parton showering. Both generators give similar results, and we show the results just of Pythia, fig. 9a. The difference at parton level is very similar to what was observed at fixed order. At hadron level without underlying event (UE) corrections, the difference remains at the level of $1-2 \%$ (though it changes sign); once one includes the underlying event contributions, the difference increases noticeably at lower $p_{t}$ — this is because the midpoint(1) algorithm receives somewhat larger UE corrections than SISCone. Since the underlying event is one of the things that is likely to change from Tevatron to LHC, in figure 9b we show similar curves for LHC kinematics. At parton level and at hadron level without the underlying event, the results are essentially the same as for the Tevatron. With the underlying event included, the impact of the missing stable cones in the midpoint algorithm reaches of the order of 10 to $15 \%$, and thus starts to become quite a significant effect. With Herwig, we find that the impact is little smaller because its underlying event is smaller than Pythia's at the LHC.

\subsubsection{Jet masses in 3 -jet events}

As well as the inclusive jet $p_{T}$ spectrum, we can also study more exclusive quantities. One example is the jet-mass spectrum in multi-jet events. Jet-masses are potentially of interest for QCD studies, particle mass measurements [34] and new physics searches, where they could be used to identify highly boosted $\mathrm{W} / \mathrm{Z} / \mathrm{H}$ bosons or top quarks produced in the decays of new heavy particles [35].

The simplest multi-jet events in which to study jet masses are 3-jet events. There, the 

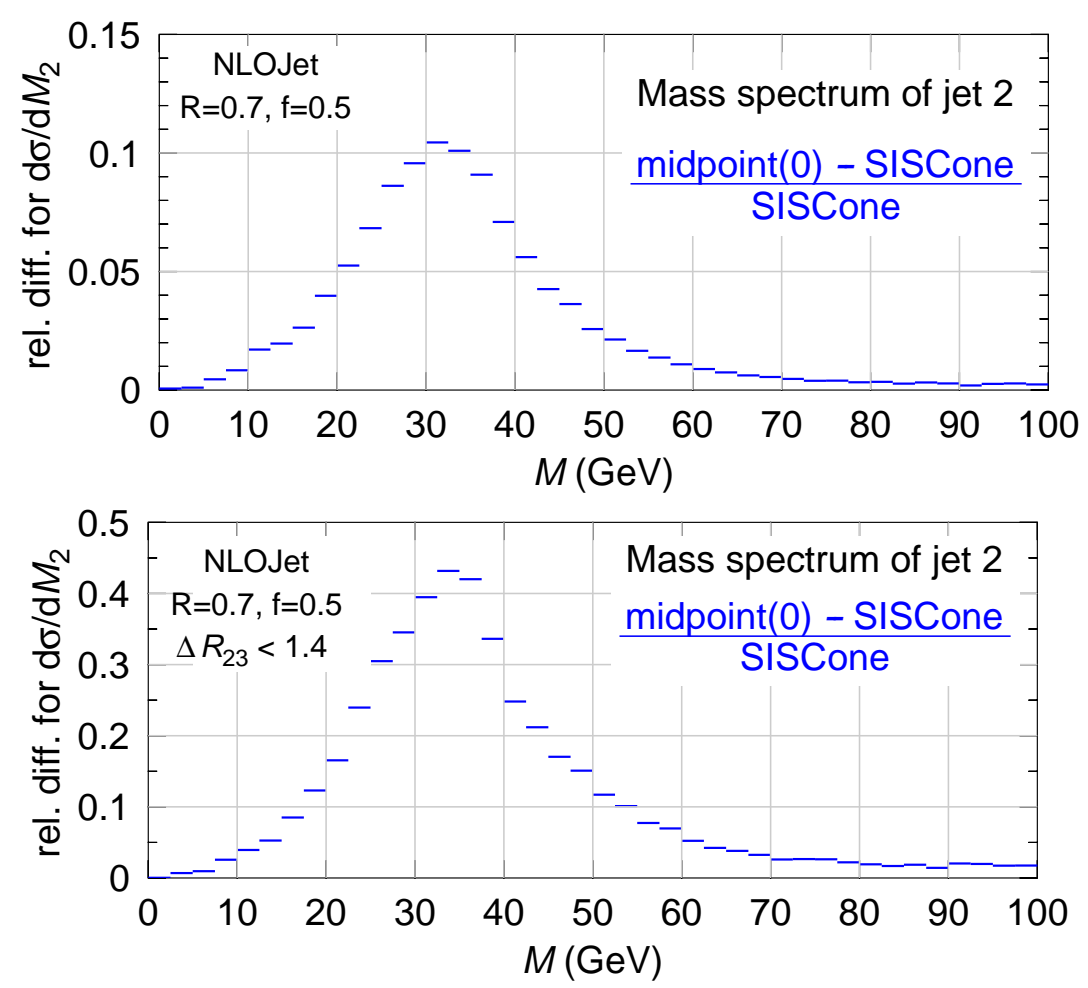

Figure 10: Mass spectrum of the second hardest jet as obtained with the different cone algorithms on tree-level 4-particle events (generated with NLOJet): the plots shows the relative difference between the midpoint and SISCone results. In the upper plot we consider all three-jet events satisfying the transverse-momentum cuts, while in the lower plot (note scale) we consider only those in which second and third jet are separated by $\Delta R_{23}<2 R$. 

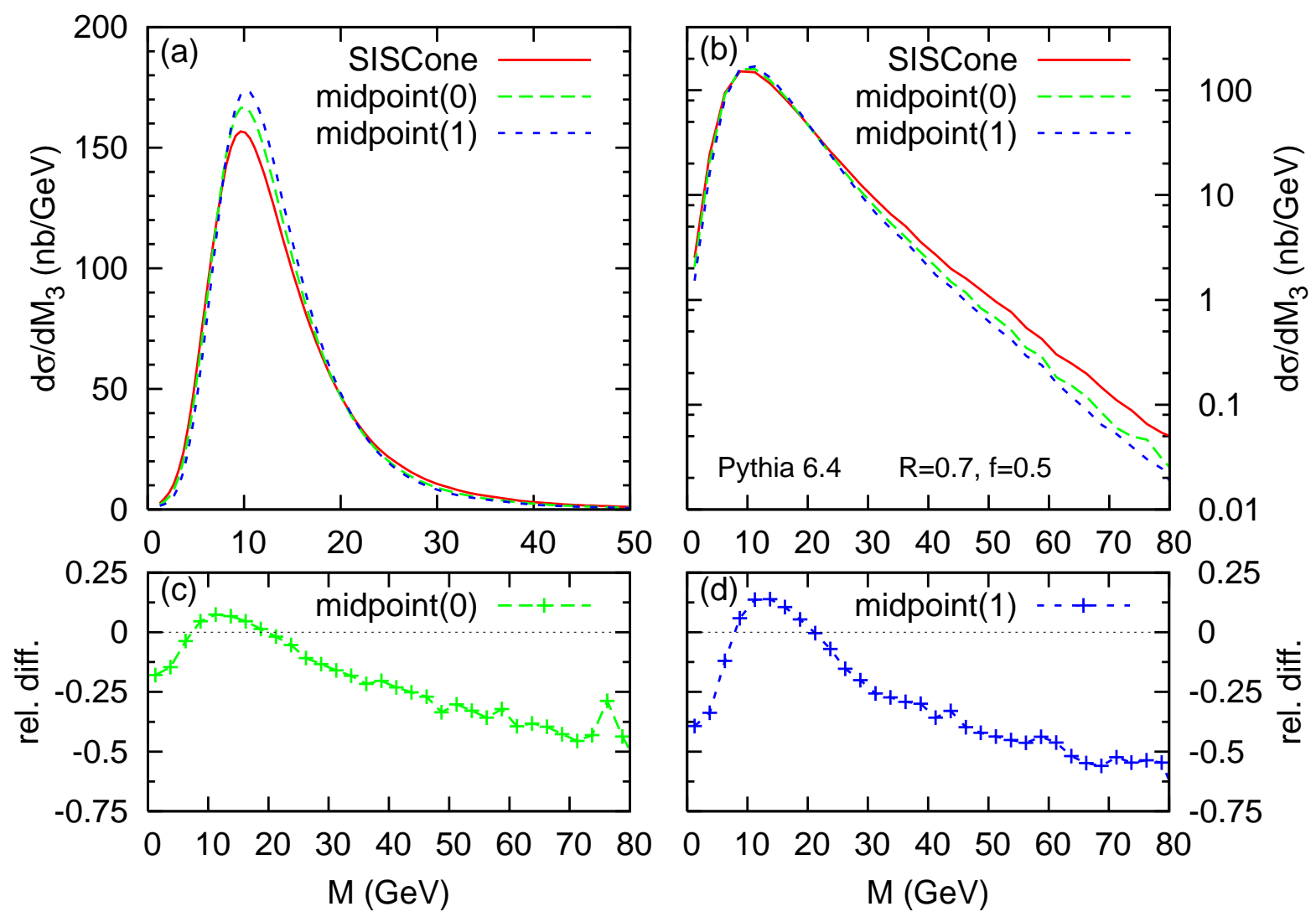

Figure 11: Mass spectrum of the third hardest jet obtained from the different cone algorithms run on three-jet Pythia events. The top-left (top-right) plot shows the spectrum in linear (logarithmic) scale and the bottom plots show the relative difference between each midpoint algorithm and SISCone. See the text for the details of the event selection. 
masses of all the jets vanish at the 3-particle level. The first order at which the jet masses become non-zero is $\mathcal{O}\left(\alpha_{s}^{4}\right)$ and this is also the order at which differences appear between the midpoint and seedless cone algorithms. Therefore, as in section 5.4.1, we generate $2 \rightarrow 4$ tree-level events, but now keep only those with exactly 3 jets with $p_{T} \geq 20 \mathrm{GeV}$ in the final state. We further impose that the hardest jet should have a $p_{T}$ of at least 120 $\mathrm{GeV}$ and the second hardest jet a $p_{T}$ of at least $60 \mathrm{GeV}$. With these cuts we can compute the jet-mass spectrum for each of the three jets and for the three different algorithms.

In the upper plot of Figure 10, we show the relative difference "(midpoint(0) - SISCone)/SISCone" for the mass spectrum of the second hardest jet. In the lower plot we show the same quantity for events in which we have placed an additional requirement that the $y-\phi$ distance between the second and third jets be less than $2 R$ (such distance cuts are often used when trying to reconstruct chains of particle decays). The midpoint algorithm's omission of certain stable cones leads to an overestimate of the mass spectrum by up to $\sim 10 \%$ without a distance cut (much smaller differences are observed for the first and third jet) and of over $40 \%$ with a distance cut. The problem is enhanced by the presence of the distance cut because many more of the selected events then have three particles in a common neighbourhood, and this is precisely the situation in which the midpoint algorithm misses stable cones ( $c f$. section 3).

We emphasise also that the NLO calculation of these mass spectra would be impossible with a midpoint algorithm, because the $10-40 \%$ tree-level differences would be converted into an infrared divergent NLO contribution.

A general comment is that the problems seen here for the midpoint algorithm without a distance cut are of the same general order of magnitude as the $16 \%$ failure rate in the IR safety tests of section 5.1, suggesting that the absolute failure rates given there are a good indicator of the degree of seriousness of issues that can arise in generic studies with the infrared unsafe algorithms.

In addition to this fixed-order parton-level analysis, we have studied the jet masses in 3-jet events at hadron level (i.e. after parton showering and hadronisation) using events generated with Pythia. At hadron level many more seeds are present, due to the large particle multiplicity. One might therefore expect the midpoint algorithm to become a good approximation to the seedless one.

For the mass of the second hardest jet, i.e. the quantity we studied at fixed order in figure 10, we find that the midpoint and seedless algorithms do give rather similar results at hadron level. In other words differences that we see in a leading order calculation are not propagated through to the full hadron level result. This is a serious practical issue for the midpoint algorithm, because a jet algorithm's principal role is to provide a good mapping between low-order parton level and hadron level.

Nevertheless, despite the many seeds that are present at hadron level, we find that there are still some observables for which the midpoint algorithm's lack of stable cones does have a large impact even at hadron level. This is the case that the mass distribution of the third hardest jet, shown in figure 11 (obtained without a distance cut) on both linear and logarithmic scales so as to help visualise the various regions of the distribution. Moderate differences are present in the peak region, but in the tail of the distribution they become 
large, up to $50 \%$. They are greater for midpoint(1) than for midpoint(0), because the seed threshold causes fewer stable cones to be found with the midpoint(1) algorithm.

These results have been checked using the Herwig Monte-Carlo. We have observed similar differences at parton-shower level, at the hadron level and at the hadron level including underlying event, both in the peak of the distribution and in the tail. We note that hadronisation corrections are substantial in the tail of the distribution, both for the midpoint and SISCone algorithms.

The above results confirm what one might naturally have expected: while very inclusive quantities may not be overly sensitive to the deficiencies of one's jet algorithm, as one extends one's investigations to more exclusive quantities, those deficiencies begin to have a much larger impact.

\section{Conclusions}

Given the widespread use of cone jet algorithms at the Tevatron and their foreseen continued use at LHC, it is crucial that they be defined in an infrared safe way. This is necessary in general so as to ensure that low-order parton-level considerations about cone jet-finding hold also for the fully showered, hadronised jets that are observed in practice. It is also a prerequisite if measurements are to be meaningfully compared to fixed order (LO, NLO, NNLO) predictions.

The midpoint iterative cone algorithm currently in use is infrared unsafe, as can be seen by examining the sets of stable cones that are found for simple three-parton configurations. This may seem surprising given that the midpoint algorithm was specifically designed to avoid an earlier infrared safety problem - however the midpoint infrared problem appears at one order higher in the coupling, and this is presumably why it was not identified in the original analyses. The tests shown in section 5.1 suggest that the midpoint-cone infrared safety problems, while smaller than without the midpoint, are actually quite significant $(\sim 15 \%)$.

We therefore advocate that where a cone jet algorithm is used, it be a seedless variant. For such a proposal to be realistic it is crucial that the seedless variant be practical. The approaches adopted in fixed order codes take $\mathcal{O}\left(N 2^{N}\right)$ time and are clearly not suitable in general. Here we have shown that it is possible to carry out exact seedless jet-finding in expected $\mathcal{O}\left(N n^{3 / 2}\right)$ time with $\mathcal{O}\left(N n^{1 / 2}\right)$ storage, or almost exactly 20 in expected $\mathcal{O}(N n \ln n)$ time with $\mathcal{O}(N n)$ storage (we recall that $N$ is the total number of particles, $n$ the typical number of particles in a jet). The second of these approaches has been implemented in a $\mathrm{C}++$ code named SISCone, available also as a plugin for the FastJet package. For $N \sim 1000$ it is comparable in speed to the existing CDF midpoint code with $1 \mathrm{GeV}$ seeds. While this is considerably slower than the $N \ln N$ and related FastJet strategies [20] for the $k_{t}$ and Cambridge/Aachen jet algorithms, it remains within the limits of usability and provides for the first time a cone algorithm that is demonstrably infrared and collinear safe at all orders, and suitable for use at parton level, hadron level and detector level.

\footnotetext{
${ }^{20}$ with a failure probability that can be made arbitrarily small and that we choose to be $\lesssim 10^{-18}$.
} 
As well as being infrared safe, a jet algorithm must provide a faithful mapping between expectations based on low-order perturbative considerations, and observations at hadron level. There has been considerable discussion of worrisome possible violations of such a correspondence for cone algorithms, the " $R_{\text {sep }}$ " issue. For SISCone we find however that the correspondence holds well.

An obvious final question is that of the impact on physics results of switching from the midpoint to the seedless cone. For inclusive quantities, one expects the seedless cone jet algorithm to give results quite similar to those of the midpoint cone, because the IR unsafety of the midpoint algorithm only appears at relatively higher orders. This is borne out in our fixed order and parton-shower studies of the inclusive jet spectrum where we see differences between the midpoint and SISCone algorithms of about a couple of percent. At moderate $p_{t}$ at hadron level, the differences can increase to $5-10 \%$, because SISCone has a lower sensitivity to the underlying event, a welcome 'fringe-benefit' of the seedless algorithm.

For less inclusive quantities, for example the distribution of jet masses in multi-jet events, differences can be significant. We find that for 3 -jet events, the absence of some stable cones (i.e. infrared unsafety) in the midpoint algorithm leads to differences compared to SISCone at the $\sim 10 \%$ level at leading order $\left(\alpha_{s}^{4}\right)$ in a large part of the jet-mass spectrum. Greater effects still, up to $50 \%$, are seen with specific cuts at fixed order, and in the tails of the jet-mass spectra for parton-shower events. Thus, even if the infrared safety issues of the midpoint algorithm appear to be at the limit of today's accuracy when examining inclusive quantities, for measurements of even moderate precision in multi-jet configurations (of increasing interest at Tevatron and omnipresent at LHC), the use of a properly defined cone algorithm such as SISCone is likely to be of prime importance.

\section{Acknowledgements}

We are grateful to Markus Wobisch for many instructive discussions about cone algorithms, Steve Ellis and Joey Huston for exchanges about their IR safety and $R_{\text {sep }}$, Matteo Cacciari for helpful suggestions on the SISCone code and Giulia Zanderighi for highlighting the question of collinear safety. We thank them all, as well as George Sterman, for useful comments and suggestions on the manuscript. We also gratefully acknowledge Mathieu Rubin for a careful reading of an early version of the manuscript, Andrea Banfi for pointing out a relevant reference and Torbjörn Sjöstrand for assistance with Pythia. The infrared unsafe configuration shown here was discovered subsequent to discussions with Mrinal Dasgupta on non-perturbative properties of cone jet algorithms. This work has been supported in part by grant ANR-05-JCJC-0046-01 from the French Agence Nationale de la Recherche. G.S. is funded by the National Funds for Scientific Research (Belgium). Finally, we thank the Galileo Galilei Institute for Theoretical Physics for hospitality and the INFN for partial support during the completion of this work. 


\section{A Further computational details}

\section{A.1 Cone multiplicities}

In evaluating the computational complexity of (computational) algorithms for various stages of the cone jet algorithm it is necessary to know the numbers of distinct cones and of stable cones. Such information also constitutes basic knowledge about cone jet definitions, which may for example be of relevance in understanding their sensitivity to pileup, i.e. multiple $p p$ interactions in the same bunch crossing.

Since large multiplicities will be due to pileup, let us consider a simple model for the event structure which mimics pileup, namely a set of momenta distributed randomly in $y$ and $\phi$ and all with similar $p_{t}$ 's (or alternatively with random $p_{t}$ 's in some limited range).

Given that the particles will be spread out over a region in $y, \phi$ that is considerably larger than the cone area, in addition to $N$, the total number of particles, it is useful to introduce also $n$, the number of points likely to be contained in a region of area $\pi R^{2}$.

The first question to investigate is that of the number of distinct cones. The number of pairs of points that has to be investigated is $\mathcal{O}(N n)$. However some of these pairs of points will lead to identical cones. It is natural to ask whether, despite this, the number of distinct cones is still $\mathcal{O}(N n)$. To answer this question, one may examine how far one can displace a cone in any given direction before its point content changes. The area swept when moving a cone a distance $\delta R$ is $4 R \delta R$, and the average number of points intersected is $4 \rho R \delta R$ where $\rho=\mathcal{O}\left(n / R^{2}\right)$ is the density of points (per unit area). Therefore the distance moved before the cone edge is likely to touch a point is $\delta R=(4 \rho R)^{-1}=\mathcal{O}(R / n)$. Correspondingly the area in which one can move the centre of cone without changing the cone's contents is $\pi(\delta R)^{2}=\mathcal{O}\left(R^{2} / n^{2}\right)$. Given that the total area is $\mathcal{O}\left(R^{2} N / n\right)$ we have that the number of distinct cones is $\mathcal{O}(N n)$, the same magnitude as the number of relevant point pairs.

Let us now consider the number of stable cones. If we take a cone at random and sum its momenta then the resulting momentum axis will differ from the original cone axis by an amount typically of order $R / \sqrt{n}$ (since the standard deviation of $y$ and $\phi$ for set of points in the cone is $\mathcal{O}(R)$ ). The probability of the difference being $\lesssim R / n$ in both the $y$ and $\phi$ directions (i.e. the probability that the new axis contains the same set of particles) is $\sim(R / n)^{2} /(R / \sqrt{n})^{2} \sim 1 / n$. Therefore the number of stable cones is $\mathcal{O}(N)$. This assumes a random distribution of particles. There may exist special classes of configurations for which the number of stable cones is greater than $\mathcal{O}(N)$. Therefore timing results that are sensitive to the number of stable cones are to be understood as "expected" results rather than rigorous upper bounds.

\section{A.2 Computational complexity of the split-merge step}

To study the computational complexity of the split-merge step, we work with the expectation that there are $\mathcal{O}(N)$ initial protojets (as discussed above) and that there will be roughly $N / n \ll N$ final jets (since there are $\mathcal{O}(n)$ particles per jet). It is reasonable to 
assume that there will be roughly equal numbers of merging and splitting operations. Splitting leaves the number of protojets unchanged, while merging reduces it by 1 . Therefore there will be $\mathcal{O}(N)$ split-merge steps before we reach the final list of jets.

There are three kinds of tasks in the split-merge procedure. Firstly one has to maintain a list of jets ordered in $\tilde{p}_{t}$, both for finding the one with highest $\tilde{p}_{t}$ and for searching through the remaining jets (in order of decreasing $\tilde{p}_{t}$ ) to find an overlapping one. Maintaining the jets in order is easily accomplished with a balanced tree (for example a priority_queue or multiset in $\mathrm{C}++$ ), at a cost of $N \ln N$ for the initial construction and $\ln N$ per update, i.e. a total of $N \ln N$, which is small compared to the remaining steps.

In examining the complexity of finding the hardest overlapping jet one needs to know the cost of comparing two jets for overlap as well as the typical number of times this will have to be done. A naive comparison of two jets takes time $n$. Using a $2 \mathrm{~d}$ tree structure such as a quadtree or $k$-d tree (as suggested also by Volobouev [17]), this can be reduced to $\sqrt{n}$. The number of jets to be compared before an overlap is found will depend on the event structure - if one assumes that jet positions are decorrelated with their $\tilde{p}_{t}$ 's, then $\mathcal{O}(N / n)$ comparisons will have to be made each time around the loop. The total cost of this will therefore be $N^{2} / \sqrt{n}\left(N^{2}\right)$ with (without) a $2 \mathrm{~d}$ tree.

Finally each merging/splitting procedure will take $\sqrt{n}(n)$ time with (without) a tree, so the total time spent merging and splitting will be $\mathcal{O}(N \sqrt{n})$ (or $\mathcal{O}(N n)$ without a tree).

The dominant step is the search for overlapping jets, which will have a total cost of $N^{2} / \sqrt{n}$ (with a sizable coefficient), or $N^{2}$ without any $2 \mathrm{~d}$ tree structures. Since in practice $N^{2}$ is smaller than the $N n \ln n$ needed to find the stable cones, here the introduction of a tree structure gives little overall advantage.

A final comment concerns memory usage: when not using any tree structures, the list of protojets and their contents requires $\mathcal{O}(N n)$ space, which is the same order of magnitude as the storage needed for identifying the set of stable cones in the first place. With a tree structure this can be reduced to $\mathcal{O}(N \sqrt{n})$.

\section{B Proof of IR safety of the SISCone algorithm}

In this appendix, we shall explicitly prove that SISCone, algorithms 1 3, is infrared safe. This means that if we run SISCone first with a set of hard particles, then with the same set of hard particles together with additional soft particles, then: (a) all jets found in the event without soft particles will be found also in the event with the soft particles; (b) any extra jets found in the event with soft particles will themselves be soft, i.e. they will not contain any of the hard particles. If either of these conditions fails in a finite region of phasespace for the hard particles, then the cancellation between (soft) real and virtual diagrams will be broken at some order of perturbation theory, leading to divergent jet cross sections.

We will first discuss the proof using a simplifying assumption: two protojets with distinct hard particle content have distinct values for the split-merge ordering variable, $\tilde{p}_{t}$. We shall then discuss subtleties associated with various ordering variables, and explain why $\tilde{p}_{t}$ is a valid choice. 


\section{B.1 General aspects of the proof}

By soft particles, we understand particles whose momenta are negligible compared to the hard ones. Specifically, for any set of hard particles $\left\{p_{1}, \ldots, p_{n}\right\}$ and any set of soft ones $\left\{\bar{p}_{1}, \ldots, \bar{p}_{m}\right\}$, we consider a limit in which all soft momenta are scaled to zero, so that they do not affect any momentum sums,

$$
\lim _{\left\{\bar{p}_{j}\right\} \rightarrow 0}\left(\sum_{i=1}^{n} p_{i}+\sum_{j=1}^{m} \bar{p}_{j}\right)=\sum_{i=1}^{n} p_{i} .
$$

In what follows, the limit of the momenta of the soft particles being taken to zero will be implicit.

Let us now compare two different runs of the cone algorithm: in the first one, referred to as the "hard event", we compute the jets starting with a list of hard particles $\left\{p_{1}, \ldots, p_{N}\right\}$, and, in the second one, referred to as the "hard+soft event", we compute the jets with the same set of hard particles plus additional soft particles $\left\{\bar{p}_{1}, \ldots, \bar{p}_{M}\right\}$. As mentioned above, the IR safety of the SISCone algorithm amounts to the statements (a) that for every jet in the hard event there is a corresponding jet in the hard+soft event with identical hard particle content (plus possible extra soft particles) and (b) that there are no hard jets in the hard+soft event that do not correspond to a jet in the hard event. To prove this, we shall proceed in two steps: first, we shall show that the determination of stable cones is IR safe, then that the split-merge procedure is also IR safe.

The IR safety of the stable-cone determination is a direct consequence of the fact that:

- each cone initially built from the hard particles only was determined by two particles in algorithm 2. This cone is thus still present when adding soft particles and, because of eq. (7), is still stable. Hence, all stable cones from the hard event are also present after inclusion of soft particles, the only difference being that they also contain extra soft particles which do not modify their momentum.

- no new stable cone containing hard particles can appear. Indeed, if a new stable cone appeared, $S_{\text {new }}$ with content $\left\{p_{\alpha_{1}}, \ldots, p_{\alpha_{n}}, \bar{p}_{\bar{\alpha}_{1}}, \ldots, \bar{p}_{\bar{\alpha}_{m}}\right\}$, then the fact that its momentum $\sum p_{\alpha_{i}}+\sum \bar{p}_{\bar{\alpha}_{j}}$ corresponds to a stable cone, implies, by eq. (7), that the cone with just the hard momenta $p_{\alpha_{i}}$ is also stable. However as shown in section 4.2 all stable cones in the hard event have already been identified, therefore this cone cannot be new.

From these two points, one can deduce that after the determination of the stable cones we end up with two different kinds of stable cones: firstly, there are those that are the same as in the hard event but with possible additional soft particles; and secondly there are stable cones that contain only soft particles. So, the 'hard content' of the stable cones has not been changed upon addition of soft particles and algorithm 2] is IR safe.

The main idea behind the proof of the IR safety of the split-merge process, algorithm 3 , is to show by induction that the hard content of the protojets evolves in the same way for 
the hard and hard+soft event. Since the hard content is the same at the beginning of the process, it will remain so all along the split-merge process which is what we want to prove.

There is however a slight complication here: when running algorithm 3 over one iteration of the loop in the hard event, we sometimes have to consider more than one iteration of the loop in the hard+soft event. As we shall shortly see, in that case, only the last of these iterations modifies the hard content of the jets and it does so in the same way as in the hard event step.

So, let us now follow the steps of algorithm 3 in parallel for the hard and hard+soft event, and show that they are equivalent as concerns the hard particles. In the following analysis, item numbers coincide with the corresponding step numbers in algorithm 3 ,

2: If $p_{t, \min }$ is non-zero, all purely soft protojets will be removed from the hard+soft event and by eq. (77) the same set of hard protojets will be removed in the hard and hard+soft event. Thus the correspondence between the hard protojets in the two events will persist independently of $p_{t, \text { min }}$.

3: In general, protojets with identical hard content will have nearly identical $\tilde{p}_{t}$ values, whereas protojets with different hard-particle content will have substantially different $\tilde{p}_{t}$ values 21 Therefore the addition of soft particles will not destroy the $\tilde{p}_{t}$ ordering and the protojet with the largest $\tilde{p}_{t}$ in the hard event, $i$ will have the same hard content as the one in the hard+soft event (let us call it $i^{\prime}$ ).

4: The selection of the highest- $\tilde{p}_{t}$ protojet $j\left(j^{\prime}\right.$ in the hard+soft case) that overlaps with $i\left(i^{\prime}\right)$ can differ in the hard and hard+soft events, and we need to consider separately the cases where this does not, or does happen. The first case, C1, is that $i^{\prime}$ and $j^{\prime}$ overlap in their hard content - because of the common $\tilde{p}_{t}$ ordering, $j^{\prime}$ must then have the same hard content as $j$. The second case, C2, is that $i^{\prime}$ and $j^{\prime}$ only overlap through their soft particles, so $j^{\prime}$ cannot be the 'same' jet as $j$ (since $j$ by definition overlaps with $i$ through hard particles). By following the remaining part of the loop, we shall show that in the first case all modifications of the hard content are the same in the hard and hard+soft events, while, for the second case, the iteration of the loop in the hard+soft event does not modify any hard content of the protojets. In this second case, we then proceed to the next iteration of the loop in the hard+soft event but stay at the same one for the hard event.

C1: The two protojets $i^{\prime}$ and $j^{\prime}$ overlap in their hard content

6]77 We need to compute the fraction of $\tilde{p}_{t}$ shared by the two protojets. Since the hard contents of $i(j)$ and $i^{\prime}\left(j^{\prime}\right)$ are identical, the fraction of overlap, given by the hard content only, will be the same in the hard and hard+soft events. Hence, the decision to split or merge the protojets will be identical.

\footnotetext{
${ }^{21}$ As mentioned already, this point is more delicate than it might seem at first sight. We come back to it in the second part of this appendix.
} 
8. Since the centres of both protojets are the same in the hard and hard+soft events, the decision to attribute a hard particle to one protojet or the other will be the same in both events. Hence splitting will reorganise hard particles in the same way for the hard+soft event as for the hard one.

10. In both the hard and the hard+soft events, the merging of the two protojets will result in a single protojet with the same hard content.

C2: The two protojets $i^{\prime}$ and $j^{\prime}$ overlap through soft particles only

6]7. Since the fraction of $\tilde{p}_{t}$ shared by the protojets will be 0 in the limit eq. (7), the two protojets will be split.

8: In the splitting, only shared particles, i.e. soft particles, will be reassigned to the first or second protojet. The hard content is therefore left untouched, as is the $\tilde{p}_{t}$ ordering of the protojets.

11: At the end of the splitting/merging of the overlapping protojets, we have to consider the two possible overlap cases separately: in the first case, the hard contents of the protojets are modified in the same way for the hard and hard+soft event. This case is thus IR safe. In the second case, the iteration of the loop in the hard+soft event does not correspond to any iteration of the loop in the hard event. However the hard content of the protojets in the hard+soft event is not modified and the $\tilde{p}_{t}$ ordering of the jets remains identical; at the next iteration of the hard+soft loop, the new $j^{\prime}$ may once again have just soft overlap with $i^{\prime}$ and the loop will thus continue iterating, splitting the soft parts of the jets, but leaving the hard content of the jets unchanged. This will continue until $j^{\prime}$ corresponds to the $j$ of the hard event, i.e. we encounter case 1.22 Therefore even though we may have gone around the loop more times in the hard+soft event, we do always reach a stage where the split-merge operation in the hard+soft event coincides with that in the hard event, and so this part of the procedure is infrared safe.

5.14: Up to possible intermediate loops involving case 2 above, when the protojet $i$ has no overlapping protojets in the hard event, the corresponding $i^{\prime}$ in the hard + soft event has no overlaps either. Final jets will thus be added one by one with the same hard content in the hard and hard+soft events.

This completes the proof that the SISCone algorithm is IR safe, modulo subtleties related to the ordering variable, as discussed below. Regarding the 'merge identical protojets' (MIP) procedure:

\footnotetext{
${ }^{22}$ Note that the second case can only happen a finite number of times between two occurrences of the first case: as the $\tilde{p}_{t}$ ordering is not modified during the second case, each time around the loop the overlap will involve a $j^{\prime}$ with a lower $\tilde{p}_{t}$ than in the previous iteration, until one reaches the $j^{\prime}$ that corresponds to $j$.
} 
12: In algorithm 3, we do not automatically merge protojets appearing with the same content during the split-merge process. This is IR safe. If instead we allow for two identical protojets to be automatically merged, then when two protojets have the same hard content but differ as a result of their soft content, they are automatically merged in the hard event but not in the hard+soft event. This in turn leads to IR unsafety of the final jets.

A final comment concerns collinear safety and cocircular points. When defining a candidate cone from a pair of points, if additional points lie on the edge of the cone, then there is an ambiguity as to whether they will be included in the cone. From the geometrical point of view, this special case of cocircular points (on a circle of radius $\mathrm{R}$ ) can be treated by considering all permutations of the the cocircular points being included or excluded from the circle contents. SISCone contains code to deal with this general issue. The case of identically collinear particles, though a specific example of cocircularity, also adds the problem that a circle cannot properly be defined from two identical points. For explicit collinear safety we thus simply merge any collinear particles into a single particle, step 1 of algorithm 2. Given the resulting collinear-safe set of protojets, the split-merge steps preserve collinear safety, since particles at identical $y-\phi$ coordinates are treated identically.

\section{B.2 Split-merge ordering variable}

Suppose we use some generic variable $v$ (which may be $p_{t}, E_{t}, m_{t}, \tilde{p}_{t}$, etc.) to decide the order in which we select protojets for the split-merge process. A crucial assumption in the proof of IR safety is that two jets with different hard content will also have substantially different values for $v$, i.e. the ordering of the $v$ 's will not be changed by soft modifications. If this is not the case then the choice of the hard protojets that enter a given split-merge loop iteration can be modified by soft momenta, with a high likelihood that the final jets will also be modified.

At first sight one might think that whatever variable is used, it will have different values for distinct hard protojets. However, momentum conservation and coincident masses of identical particles can introduce relations between the kinematic characteristics of distinct protojets. Some care is therefore needed so as to ensure that these relations do not lead to degeneracies in the ordering, with consequent ambiguities and infrared unsafety for the final jets. In particular:

- Two protojets can have equal and opposite transverse momenta if between them they contain all particles in the event (and the event has no missing energy or 'ignored' particles such as isolated leptons). It is probably fair to assume that no two protojets will have identical longitudinal components, since in $p p$ collisions the hard partonic reaction does not occur in the $p p$ centre of mass frame.

- Two protojets will have identical masses if they each stem exclusively from the same kind of massive particle. The two massive particles may be undecayed (e.g. fully reconstructed $b$-hadrons) or decayed (top, $W, Z, H$, or some non-standard new 
particle), or even one decayed and the other not (some hypothetical particle with a long lifetime) 23 In the second case we can assume that two identical decayed particles have different decay planes, because there is a vanishing phase space for them to have identical decay planes.

Note that in a simple two-parton event almost any choice of variable will lead to a degeneracy (no sensible invariant will distinguish the two particles), however this specific case is not problematic because for $R<\pi / 2$ neither of the two partons can be in a protojet that overlaps with anything else. From the point of view of IR safety, it is only for 'fat' (non-collimated) hard protojets that we need worry about the problem of degeneracies in the split-merge ordering, because only then will there be overlaps whose resolution is ambiguous in the presence of degeneracies.

Let us now consider what occurs with various possible choices for the split-merge variable.

$p_{t}$ : This choice, adopted in certain codes [13, 19], can be seen to have a problem for events with momentum conservation in the hadronic part, because if two non-overlapping protojets contain, between them, all the hard particles then they will have identical $p_{t}$ 's. If they each overlap with a common third protojet, the resulting split-merge sequence will be ambiguous. Table 4 provides an example of such an event. The simplest occurrences of this problem $(4 h+1 s)$ apply only to $R>\pi / 4$ (four particles must form at least 3 fat protojets). The problem arises also for smaller $R$ values, but only at higher multiplicities.

$m_{t}$ : A workaround for the event of table 4 is to use the transverse mass, $m_{t}=\sqrt{p_{t}^{2}+m^{2}}$. In pure QCD, with all particles stable, this is a good variable, because even if two fat protojets have identical $p_{t}$ 's through momentum conservation, the fact that they are 'fat' implies that they will be massive (over and above intrinsic particle masses), and the phase space for them to have identical masses vanishes, thus killing any IR divergences. However, for events with two identical decaying particles, two fat protojets resulting from the particle decays can have identical $p_{t}$ 's (by momentum conservation) and identical masses (because the decaying particles were identical). This could happen for example in the fully hadronic decay channel for $t \bar{t}$ events. Thus, this choice is not advisable in a general purpose algorithm.

$E_{t}$ : The variable used in the original run II proposal was $E_{t}[\underline{6}$. It has the drawback that it is not longitudinally boost invariant: at central rapidity it is equal to $m_{t}$, while at high rapidities it tends to $p_{t}$. Because the phase space for two protojets to have identical rapidities vanishes (recall that we do not fix the partonic centre-of-mass), two protojets with identical $p_{t}$ 's and masses will have different $E_{t}$ 's, because the

\footnotetext{
${ }^{23}$ Strictly speaking, for all scenarios of decayed heavy particles, the finite width $\Gamma$ of the particle ensures that the two jets actually have slightly different masses, breaking any degeneracies. In practice however, $\Gamma_{W, Z, t} \sim 1 \mathrm{GeV}$ and (for a light Higgs) $\Gamma_{H} \ll \Lambda_{Q C D}$, whereas for the width to save us from the dangers of degeneracies we would need $\Gamma \gg \Lambda_{Q C D}$.
} 
event 1

\begin{tabular}{r|rrr}
\hline $\mathrm{n}$ & $p_{x}$ & $p_{y}$ & $p_{z}$ \\
\hline 0 & 86.01 & 66 & 0 \\
1 & 64 & -66 & 0 \\
2 & -77 & -70 & 0 \\
3 & -73 & 70 & 0 \\
4 & -0.01 & 0 & 2
\end{tabular}

event 2

\begin{tabular}{r|rrr}
\hline $\mathrm{n}$ & $p_{x}$ & $p_{y}$ & $p_{z}$ \\
\hline 0 & 85.99 & 66 & 0 \\
1 & 64 & -66 & 0 \\
2 & -77 & -70 & 0 \\
3 & -73 & 70 & 0 \\
4 & 0.01 & 0 & 2
\end{tabular}

Table 4: Illustration of two events that conserve transverse momentum and differ only through a soft particle, but lead to different hard jets with a split-merge procedures that uses $p_{t}$ as the ordering variable and for measuring overlap. All the particles are to be taken massless. For $R=0.9$ and $f=0.7$ each event has stable cones consisting of $\{01\},\{23\}$ and $\{12\}$, as well as all single particles. The slight difference in momenta between the two events, to balance the soft particle, causes the $\{01\}(\{23\})$ protojet to have the largest $p_{t}$ in the first (second) event, it splits with $\{12\}$ (merges with $\{12\}$ ), leading after further split-merge steps to two hard jets, $\{01\}$ and $\{23\}$ (one hard 'monster' jet, $\{0123\}$ ).

degree of 'interpolation' between between $p_{t}$ and $m_{t}$ will be different. This resolves the degeneracy and should cure the resulting IR safety issue, albeit at the expense of introducing boost-dependence.

$\tilde{p}_{t}$ : The scalar sum of transverse momenta of the protojet constituents, $\tilde{p}_{t}$, has the property that it is equal to $m_{t}$ if all particles in the protojet have identical rapidities, while it is equal to $p_{t}$ (i.e. the vector sum) if all particles have identical azimuths. For a decayed massive particle, it essentially interpolates between $p_{t}$ and $m_{t}$ according to the orientation of the decay plane. The phase space for all particles to have identical azimuths vanishes, as does the phase space for the decay products of two heavy particles to have identically oriented decay planes. Therefore this choice resolves any degeneracies, as is needed for infrared safety. Another advantage of $\tilde{p}_{t}$ is that adding a particle to a protojet always increases its $\tilde{p}_{t}$ (this is not the case for $p_{t}$ or $E_{t}$ ), ensuring that the degree of overlap between a pair of jets is always bounded by 1 . Since it is also boost invariant, it is the choice that we recommend and that we adopt as our default 24

Note that the above considerations hold for any split-merge procedure that relies on ordering the jets according to a single-jet variable. One might also consider ordering according to variables determined from pairs of protojets: e.g. first split-merge the pair of protojets with the largest (or alternatively smallest) overlap, recalculate all overlaps, and then repeat until there are no further overlaps. However this specific example would also be dangerous,

\footnotetext{
${ }^{24}$ One might worry about the naturalness of a variable that depends on the decay plane of heavy particles - however, any unnaturalness is present anyway in the split-merge procedure since if two particles decay purely in the transverse plane then there is a likelihood of having overlapping protojets, whereas if they decay in longitudinally oriented decay planes they will not overlap.
} 
since the particles that are common to protojets $a$ and $b$ (say) could also be the particles that are common between $a$ and $c$, once again leading to an ambiguous split-merge sequence. One protojet-pair ordering variable that might be free of this problem is the $y-\phi$ distance between the protojets, however we have not investigated it in detail.

A final comment concerns the impact of the split-merge procedure on non-global [36] resummations for jets [37], in which one is interested in determining which of a set of ordered soft particles are in a given hard jet. A soft and collinear splitting inside the jet can modify the $\tilde{p}_{t}$ (or $E_{t}$ or $m_{t}$ ) of the jet by an amount of the same order of magnitude as a soft, large-angle emission near the edge of the jet. In events with two back-to-back narrow jets, for which there is a near degeneracy between the $\tilde{p}_{t}$ 's of the two hard jets, this can affect which of the two hard protojets split-merges first with an overlapping soft protojet, leading to ambiguities in the assignment of the soft particles to the two hard jets. This interaction between collinear and soft modes is somewhat reminiscent of that in [38], though the origin and structure are kinematical in our case. Considering only branchings with transverse momenta above $\epsilon p_{t \text {,hard }}$, for $R>\pi / 4$ this is likely to be relevant in events with two equally soft particles $\left(\alpha_{s}^{2} \ln \epsilon\right)$ and $n$ soft-collinear splittings $\left(\alpha_{s}^{n} \ln ^{2 n} \epsilon\right)$ giving an overall contribution $\alpha_{s}^{n+2} \ln ^{2 n+1} \epsilon$. This competes with the normal soft-ordered non-global logarithms, starting from order $\alpha_{s}^{3} \ln ^{3} \epsilon$. For $R \leq \pi / 4$, the problem will only arise with a greater number of equally soft large-angle particles, and so will be further suppressed by powers of $\alpha_{s}$.

\section{References}

[1] TeV4LHC QCD Working Group et al., hep-ph/0610012.

[2] S. Catani, Y. L. Dokshitzer, M. H. Seymour and B. R. Webber, Nucl. Phys. B 406 (1993) 187; S. D. Ellis and D. E. Soper, Phys. Rev. D 48 (1993) 3160 hep-ph/9305266.

[3] Y. L. Dokshitzer, G. D. Leder, S. Moretti and B. R. Webber, JHEP 9708, 001 (1997) hep-ph/9707323; ; M. Wobisch and T. Wengler, hep-ph/9907280; M. Wobisch, "Measurement and QCD analysis of jet cross sections in deep-inelastic positron proton collisions at $\sqrt{s}=300 \mathrm{GeV}, "$ DESY-THESIS-2000-049.

[4] V. M. Abazov et al. [D0 Collaboration], Phys. Lett. B 525 (2002) 211 hep-ex/0109041.

[5] A. Abulencia et al. [CDF II Collaboration], Phys. Rev. Lett. 96 (2006) 122001 hep-ex/0512062.

[6] G. C. Blazey et al., hep-ex/0005012.

[7] M. H. Seymour and C. Tevlin, JHEP 0611 (2006) 052 [hep-ph/0609100].

[8] G. Sterman and S. Weinberg, Phys. Rev. Lett. 39 (1977) 1436. 
[9] S.D. Ellis, private communication to the OPAL Collaboration; D.E. Soper and H.-C. Yang, private communication to the OPAL Collaboration; L.A. del Pozo, University of Cambridge PhD thesis, RALT-002, 1993; R. Akers et al. [OPAL Collaboration], Z. Phys. C 63, 197 (1994); M. H. Seymour, Nucl. Phys. B 513 (1998) 269 hep-ph/9707338.

[10] V. Abazov et al. [D0 Collaboration], hep-ex/0608052.

[11] A. Abulencia et al. [CDF Run II Collaboration], hep-ex/0512020.

[12] L. A. del Pozo and M. H. Seymour, pxcone (unpublished code).

[13] The CDF Collaboration's implementation of the Tevatron Run-II cone definition [6] is available at http://www.pa.msu.edu/ huston/Les_Houches_2005/Les_Houches_SM.html

[14] G. Arnison et al. [UA1 Collaboration], Phys. Lett. B 132 (1983) 214.

[15] J. E. Huth et al., in Snowmass Summer Study (1990) pp. 134-136.

[16] N. Kidonakis, G. Oderda and G. Sterman, Nucl. Phys. B 525 (1998) 299 hep-ph/9801268.

[17] I. Volobouev, presentation at MC4LHC meeting, CERN, July 2006.

[18] J. Campbell and R. K. Ellis, Phys. Rev. D 65 (2002) 113007 hep-ph/0202176.

[19] Z. Nagy, Phys. Rev. Lett. 88 (2002) 122003 [hep-ph/0110315]; Phys. Rev. D 68 (2003) 094002 hep-ph/0307268.

[20] M. Cacciari and G. P. Salam, Phys. Lett. B 641 (2006) 57 hep-ph/0512210.

[21] S. D. Ellis, J. Huston and M. Tonnesmann, in Proc. of the APS/DPF/DPB Summer Study on the Future of Particle Physics (Snowmass 2001) ed. N. Graf, p. P513 hep-ph/0111434.

[22] M. Luscher, Comput. Phys. Commun. 79 (1994) 100 hep-lat/9309020.

[23] H. Samet, ACM Computing Surveys (CSUR) 16 (1984) 187.

[24] J. L. Bentley, Communications of the ACM 18 (1975) 509.

[25] A. Banfi, G. P. Salam and G. Zanderighi, JHEP 0503 (2005) 073 hep-ph/0407286; Phys. Lett. B 584 (2004) 298 hep-ph/0304148.

[26] T. Sjostrand et al., Comput. Phys. Commun. 135, 238 (2001) hep-ph/0010017; T. Sjostrand et al., hep-ph/0308153. 
[27] J. M. Campbell, J. W. Huston and W. J. Stirling, Rept. Prog. Phys. 70 (2007) 89 hep-ph/0611148.

[28] F. Abe et al. [CDF Collaboration], Phys. Rev. D 45 (1992) 1448.

[29] B. Abbott, M. Bhattacharjee, D. Elvira, F. Nang and H. Weerts [for the D0 Collaboration], FERMILAB-PUB-97-242-E.

[30] S. D. Ellis, Z. Kunszt and D. E. Soper, Phys. Rev. Lett. 69 (1992) 3615 [hep-ph/9208249].

[31] G. Marchesini, B. R. Webber, G. Abbiendi, I. G. Knowles, M. H. Seymour and L. Stanco, Comput. Phys. Commun. 67 (1992) 465; G. Corcella et al., JHEP 0101 (2001) 010 hep-ph/0011363.

[32] S. Catani and B. R. Webber, JHEP 9710 (1997) 005 hep-ph/9710333].

[33] A. Daleo, T. Gehrmann and D. Maitre, hep-ph/0612257.

[34] S. Fleming, A. H. Hoang, S. Mantry and I. W. Stewart, hep-ph/0703207; A. Hoang and S. Mantry, presentations at the "Ringberg workshop on non-perturbative QCD of jets", Ringberg Castle, 8-10 January 2007.

[35] J. Huston, private communication; A. L. Fitzpatrick, J. Kaplan, L. Randall and L. T. Wang, hep-ph/0701150; B. Lillie, L. Randall and L. T. Wang, hep-ph/0701166. W. Skiba and D. Tucker-Smith, hep-ph/0701247; B. Holdom, hep-ph/0702037; J. M. Butterworth, J. R. Ellis and A. R. Raklev, hep-ph/0702150.

[36] M. Dasgupta and G. P. Salam, Phys. Lett. B 512 (2001) 323 [hep-ph/0104277], JHEP 0203 (2002) 017 [hep-ph/0203009]; A. Banfi, G. Marchesini and G. Smye, JHEP 0208 (2002) 006 hep-ph/0206076].

[37] R. B. Appleby and M. H. Seymour, JHEP 0212 (2002) 063 [hep-ph/0211426]; A. Banfi and M. Dasgupta, Phys. Lett. B 628 (2005) 49 (hep-ph/0508159]; Y. Delenda, R. Appleby, M. Dasgupta and A. Banfi, JHEP 0612 (2006) 044 [hep-ph/0610242].

[38] J. R. Forshaw, A. Kyrieleis and M. H. Seymour, JHEP 0608 (2006) 059 hep-ph/0604094. 